\title{
Invariant Fibrations for some Birational Maps of $\mathbb{C}^{2 *}$
}

\author{
Anna Cima ${ }^{(1)}$ and Sundus Zafar ${ }^{(1)}$ \\ (1) Departament de Matemàtiques, Facultat de Ciències, \\ Universitat Autònoma de Barcelona, \\ 08193 Bellaterra, Barcelona, Spain \\ cima@mat.uab.cat, sundus@mat.uab.cat
}

\begin{abstract}
In this article we extract and study the zero entropy subfamilies of a certain family of birational maps of the plane. We find these zero entropy mappings and give the invariant fibrations associated to them.
\end{abstract}

Mathematics Subject Classification 2010: 14E05, 26C15, 34K19, 37B40, 37C15, 39A23.

Keywords: Birational maps, Algebraic entropy, First Integrals, Fibrations, Blowing-up, Integrability, Periodicity.

\section{Introduction}

A mapping $f=\left(f_{1}, f_{2}\right): \mathbb{C}^{2} \longrightarrow \mathbb{C}^{2}$ is said to be rational if each coordinate function is rational, that is, $f_{i}$ is a quotient of polynomials for $i=1,2$. These maps can be naturally extended to the projective plane $P \mathbb{C}^{2}$ by considering the embedding $\left(x_{1}, x_{2}\right) \in \mathbb{C} \rightarrow\left[1: x_{1}\right.$ : $\left.x_{2}\right] \in P \mathbb{C}^{2}$. The induced mapping $F: P \mathbb{C}^{2} \longrightarrow P \mathbb{C}^{2}$ has three components $F_{i}\left[x_{0}: x_{1}: x_{2}\right]$ which are homogeneous polynomials of the same degree. If $F_{1}, F_{2}, F_{3}$ have no common factors and have degree $d$, we say that $f$ or $F$ has degree $d$. Similarly we can define the degree of $F^{n}=F \circ \cdots \circ F$ for each $n \in \mathbb{N}$.

We are interested in birational maps. It is said that a rational mapping $f: \mathbb{C}^{2} \longrightarrow \mathbb{C}^{2}$ is birational if it exists an algebraic curve and another rational map $g$ such that $f \circ g=g \circ f=i d$ in $\mathbb{C}^{2} \backslash V$

The study of the dynamics generated by birational mappings in the plane has been growing in recent years, see for instance $[2,3,6,8,13,15,16,17,18,19,20,21]$.

\footnotetext{
${ }^{*}$ Acknowledgements. The authors are supported by Ministry of Economy and Competitiveness of the Spanish Government through grants MTM2013-40998-P. They are also supported by the grant 2014-SGR568 from AGAUR, Generalitat de Catalunya.
} 
It can be seen that if $f\left(x_{1}, x_{2}\right)$ is a birational map, then the sequence of the degrees of $F^{n}$ satisfies a homogeneous linear recurrence with constant coefficients (see [12] for instance). This is governed by the characteristic polynomial $\mathcal{X}(x)$ of a certain matrix associated to $F$. The other information we get from $\mathcal{X}(x)$ is the dynamical degree, $\delta(F)$, which is defined as

$$
\delta(F):=\lim _{n \rightarrow \infty}\left(\operatorname{deg}\left(F^{n}\right)\right)^{\frac{1}{n}} .
$$

The logarithm of this quantity has been called the algebraic entropy of $F$. It is known that the algebraic entropy is an upper bound of the topological entropy, which in turn is a dynamic measure of the complexity of the mapping. For instance, periodic or integrable birational mappings have zero algebraic entropy.

Birational mappings with zero algebraic entropy have been characterized, see [12] and [4]. From its results we know the existence of some fibrations associated to the mapping, which give almost a complete dynamical information of the mapping.

In this paper we consider the family of fractional maps $f: \mathbb{C}^{2} \rightarrow \mathbb{C}^{2}$ :

$$
f(x, y)=\left(\alpha_{0}+\alpha_{1} x+\alpha_{2} y, \frac{\beta_{0}+\beta_{1} x+\beta_{2} y}{\gamma_{0}+\gamma_{2} y}\right), \alpha_{1} \neq 0, \beta_{1} \neq 0, \gamma_{2} \neq 0 .
$$

This family is part of a more general family studied in [10] and [11], which in turn is a generalization of the birational mappings studied by Bedford and Kim in [2]. The goal of this paper is to extract, under affine equivalence, all mappings of type (2) having zero algebraic entropy and give the corresponding invariant fibrations associated to them.

The methodology involves the implementation of the blowing-up technique and the extension of the mappings at the Picard group (see Section 2).

In general, given a parametric family of mappings, to decide for which values of the parameters the mappings are periodic, is not an easy problem (see [9], [5], for instance). When the mapping is a plane birational mapping it is possible to face that problem (see [2]) and it is fascinating to see how these cases arise, and not only the periodic ones, also all the zero entropy cases.

The paper is organized as follows. In Section 2 we give some preliminary results and we explain how we proceed to find the invariant fibrations associated to zero entropy maps. Section 3 deals with the subfamily $\alpha_{2} \neq 0$. The main result is Theorem 11. Similarly in Section 4 we consider the subfamily $\alpha_{2}=0$ getting Theorem 13 .

\section{Preliminary results}

Rational mappings $F: P \mathbb{C}^{2} \rightarrow P \mathbb{C}^{2}$ have an indeterminacy set $\mathcal{I}(F)$ of points where $F$ is ill-defined as a continuous map. This set is given by:

$$
\left.\mathcal{I}(F)=\left\{\left[x_{0}: x_{1}: x_{2}\right] \in P \mathbb{C}^{2}: F_{1}\left[x_{0}: x_{1}: x_{2}\right]=0, F_{2}\left[x_{0}: x_{1}: x_{2}\right]=0, F_{3}\left[x_{0}: x_{1}: x_{2}\right]=0\right]\right\} .
$$


If $F$ is birational then we can also consider the indeterminacy points of its inverse $F^{-1}$. On the other hand, if we consider one irreducible component $V$ of the determinant of the Jacobian of $F$, it is known (see Proposition 3.3 in [13]) that $F(V)$ reduces to a point which belongs to $\mathcal{I}\left(F^{-1}\right)$. The set of these curves which are sent to a single point is called the exceptional locus of $F$ and it is denoted by $\mathcal{E}(F)$.

It is known that the dynamical degree depends on the orbits of the indeterminacy points of the inverse of $F$ under the action of $F$, see $[12,14]$. Indeed, the key point is whether the iterates of such points coincide with any of the indeterminacy points of $F$. When it happens, this orbit is finite.

Sometimes some orbit collision appears. The expression orbit collision refers to the following: Let $S \in \mathcal{E}(F)$ which collapses at the point $A \in \mathcal{I}\left(F^{-1}\right)$ (we will write $S \rightarrow A$ to describe this behaviour). Following the orbit of $A$, assume that it ends at a point $O \in \mathcal{I}(F)$. It can happen that

$$
S \rightarrow A \rightarrow * \rightarrow \cdots \rightarrow \sigma \rightarrow \cdots \rightarrow O, \sigma \in \bar{S} \in \mathcal{E}(F) .
$$

Then, being $f$ birational, (see [12]) it exists $\bar{A} \in \mathcal{I}\left(F^{-1}\right)$ with $\bar{S} \rightarrow \bar{A}$. When it happens it is said that the orbits of $A$ and $\bar{A}$ collides. This is exactly the behaviour that we get in family (1) and what makes the family so interesting.

\subsection{Blow-up's and the Picard group}

Given a point $p \in \mathbb{C}^{2}$, let $(X, \pi)$, be the blowing-up of $\mathbb{C}^{2}$ at the point $p$. Then,translating $p$ at the origin,

$$
\pi^{-1} p=\pi^{-1}(0,0)=\{((0,0),[u: v])\}:=E_{p} \simeq P \mathbb{C}^{1}
$$

and if $q=(x, y) \neq(0,0)$, then

$$
\pi^{-1} q=\pi^{-1}(x, y)=((x, y),[x: y]) \in X .
$$

Given the point $((0,0),[u: v]) \in E_{p}$ (resp. $\left.((x, y),[x: y])\right)$ we are going to represent it by $[u: v]_{E_{p}}$ (resp. by $(x, y) \in \mathbb{C}^{2}$ or by $[1: x: y] \in P \mathbb{C}^{2}$ if it is convenient). After every blow up we get a new expanded space $X$ and the induced map $\tilde{F}: X \rightarrow X$. And then $\tilde{F}$ induces a morphism of groups, $\tilde{F}^{*}: \mathcal{P} i c(X) \rightarrow \mathcal{P} i c(X)$ just by taking classes of preimages, where $\mathcal{P} i c(X)$ is the Picard group of $X$ (see $[1,2]$ ). It is proved that after a finite number of blowing-up's we get a map $\tilde{F}$ which satisfies $\left(\tilde{F}^{n}\right)^{*}=\left(\tilde{F}^{*}\right)^{n}$. Maps $\tilde{F}$ satisfying this equality are called Algebraically Stable Maps (AS for short), (see [12]). The characteristic polynomial of the matrix of $\tilde{F}^{*}$ is the one associated to the sequence of degrees $d_{n}:=$ degree $F^{n}$. 


\subsection{Lists of orbits.}

We derive our results by using Theorem 1 below, established and proved in [1,2]. The proof of that is based in the same tools explained in the above paragraph. In order to determine the matrix of the extended map in the Picard group, it is necessary to distinguish between different behaviors of the iterates of the map on the indeterminacy points of its inverse.

The theorem is written for a general family $G$ of quadratic maps of the form $G=L \circ J$. As we will see the maps of family (18), when the triangle is non-degenerate, are linearly conjugated to such a maps. Here $L$ is an invertible linear map and $J$ is the involution in $P \mathbb{C}^{2}$ as follows:

$$
J\left[x_{0}: x_{1}: x_{2}\right]=\left[x_{1} x_{2}: x_{0} x_{2}: x_{0} x_{1}\right] .
$$

We find that the involution $J$ has an indeterminacy locus $\mathcal{I}=\left\{\epsilon_{0}, \epsilon_{1}, \epsilon_{2}\right\}$ and a set of exceptional curves $\mathcal{E}=\left\{\Sigma_{0}, \Sigma_{1}, \Sigma_{2}\right\}$, where $\Sigma_{i}=\left\{x_{i}=0\right\}$ for $i=0,1,2$, and $\epsilon_{i}=\Sigma_{j} \bigcap \Sigma_{k}$ with $\{i, j, k\}=\{0,1,2\}$ and $i \neq j \neq k, i \neq k$. Let $\mathcal{I}\left(G^{-1}\right):=\left\{a_{0}, a_{1}, a_{2}\right\}$, the elements of this set are determined by $a_{i}:=G\left(\Sigma_{i}-\mathcal{I}(J)\right)=L \epsilon_{i}$ for $i=0,1,2$; see [1].

To follow the orbits of the points of $\mathcal{I}\left(G^{-1}\right)$ we need to understand the following definitions and construction of lists of orbits in order to apply the result of Theorem 1.

We assemble the orbit of a point $p \in P \mathbb{C}^{2}$ under the map $G$ as follows. For a point $p \in \mathcal{E}(G) \cup \mathcal{I}(G)$ we say that the orbit $\mathcal{O}(p)=\{p\}$. Now consider that there exits a $p \in P \mathbb{C}^{2}$ such that its $n^{\text {th }}$ - iterate belongs to $\mathcal{E}(G) \cup \mathcal{I}(G)$ for some $n$, whereas all the other $n-1$ iterates of $p$ under $G$ are never in $\mathcal{E}(G) \cup \mathcal{I}(G)$. This is to say that for some $n$ the orbit of $p$ reaches an exceptional curve of $G$ or an indeterminacy point of $G$. We thus define the orbit of $p$ as $\mathcal{O}(p)=\left\{p, G(p), \ldots, G^{n}(p)\right\}$ and we call it a singular orbit. If for some $p \in P \mathbb{C}^{2}$ in turns out that $p$ and all of its iterates under $G$ are never in $\mathcal{E}(G) \cup \mathcal{I}(G)$ for all $n$, we set as $\mathcal{O}(p)=\left\{p, G(p), G^{2}(p) \ldots\right\}$ and $\mathcal{O}(p)$ is non singular orbit. We now make another characterization of these orbits. Consider that a singular orbit reaches an indeterminacy point of $G$, this is to say that $G^{n}(p) \in \mathcal{I}(G)$ but its not in $\mathcal{E}(G)$. We call such orbits as singular elementary orbits and we refer them as SE-orbits. To apply Theorem 1 we need to organize our SE orbits into lists in the following way.

Two orbits $\mathcal{O}_{1}=\left\{a_{1}, \ldots, \epsilon_{j_{1}}\right\}$ and $\mathcal{O}_{2}=\left\{a_{2}, \ldots, \epsilon_{j_{2}}\right\}$ are in the same list if either $j_{1}=2$ or $j_{2}=1$, that is, if the ending index of one orbit is the same as the beginning index of the other. We say that a list of orbits

$$
\mathcal{L}=\left\{\mathcal{O}_{i}=\left\{a_{i}, \ldots, \epsilon_{\tau(i)}\right\}, \ldots, \mathcal{O}_{j}=\left\{a_{j}, \ldots, \epsilon_{\tau(j)}\right\}\right\}
$$


is closed if $\tau(j)=i$. Otherwise it is an open list. For instance,

$$
\begin{aligned}
& \mathcal{L}_{1}=\left\{\mathcal{O}_{1}=\left\{a_{1}, \ldots, \epsilon_{1}\right\}\right\} \\
& \mathcal{L}_{2}=\left\{\mathcal{O}_{0}=\left\{a_{0}, \ldots, \epsilon_{2}\right\}, \mathcal{O}_{2}=\left\{a_{2}, \ldots, \epsilon_{0}\right\}\right\} \\
& \mathcal{L}_{3}=\left\{\mathcal{O}_{0}=\left\{a_{0}, \ldots, \epsilon_{1}\right\}, \mathcal{O}_{1}=\left\{a_{1}, \ldots, \epsilon_{2}\right\}, \mathcal{O}_{2}=\left\{a_{2}, \ldots, \epsilon_{0}\right\}\right\}
\end{aligned}
$$

are closed lists.

We now define two polynomials $\mathcal{T}_{\mathcal{L}}$ and $\mathcal{S}_{\mathcal{L}}$ which we will use to state Theorem 1 . Let $n_{i}$ denote the sum of the number of elements of an orbit $\mathcal{O}_{i}$ and let $\mathcal{N}_{\mathcal{L}}=n_{u}+\ldots+n_{u+\mu}$ denote the sum of the numbers of elements of each list $|\mathcal{L}|$. If $\mathcal{L}$ is closed then $\mathcal{T}_{\mathcal{L}}=x^{\mathcal{N}_{\mathcal{L}}}-1$ and if $\mathcal{L}$ is open then $\mathcal{T}_{\mathcal{L}}=x^{\mathcal{N}_{\mathcal{L}}}$. Now we define $\mathcal{S}_{\mathcal{L}}$ for different lists as follows:

$$
\mathcal{S}_{\mathcal{L}}(x)=\left\{\begin{array}{cc}
1 & \text { if }|\mathcal{L}|=\left\{n_{1}\right\}, \\
x^{n_{1}}+x^{n_{2}}+2 & \text { if } \mathcal{L} \text { is closed and }|\mathcal{L}|=\left\{n_{1}, n_{2}\right\}, \\
x^{n_{1}}+x^{n_{2}}+1 & \text { if } \mathcal{L} \text { is open and }|\mathcal{L}|=\left\{n_{1}, n_{2}\right\}, \\
\sum_{i=1}^{3}\left[x^{\mathcal{N}_{\mathcal{L}}-n_{i}}+x^{n_{i}}\right]+3 & \text { if } \mathcal{L} \text { is closed and }|\mathcal{L}|=\left\{n_{1}, n_{2}, n_{3}\right\}, \\
\sum_{i=1}^{3} x^{\mathcal{N}_{\mathcal{L}}-n_{i}}+\sum_{i \neq 2} x^{n_{i}}+1 & \text { if } \mathcal{L} \text { is open and }|\mathcal{L}|=\left\{n_{1}, n_{2}, n_{3}\right\} .
\end{array}\right.
$$

Theorem 1. ([2]) If $G=L \circ J$, then the dynamical degree $\delta(G)$ is the largest real zero of the polynomial

$$
\mathcal{X}(x)=(x-2) \prod_{\mathcal{L} \in \mathcal{L}^{c} \cup \mathcal{L}^{o}} \mathcal{T}_{\mathcal{L}}(x)+(x-1) \sum_{\mathcal{L} \in \mathcal{L}^{c} \cup \mathcal{L}^{o}} S_{L}(x) \prod_{\mathcal{L}^{\prime} \neq \mathcal{L}} \mathcal{T}_{\mathcal{L}^{\prime}}(x) .
$$

Here $\mathcal{L}$ runs over all the orbit lists.

This theorem enables us to calculate the characteristic polynomial associated to $d_{n}$. To this end we have to perform the lists of the orbits of the points in $\mathcal{I}\left(F^{-1}\right)$, but for this we have to do the necessary blow-up's to get an AS mapping.

In order to get AS maps we will use the following useful result showed by Fornaess and Sibony in [14] (see also Theorem 1.14) of [12]:

The map $\tilde{F}$ is AS if and only if for every exceptional curve $C$ and all $n \geq 0, \tilde{F}^{n}(C) \notin \mathcal{I}(\tilde{F})$.

\subsection{Zero entropy}

The following result is quiet useful in our work. It is a direct consequence of Theorem 0.2 of [12]. Given a birational map $F$ of $P \mathbb{C}^{2}$, let $\tilde{F}$ be its regularized map so that the induced map $\tilde{F}^{*}: \mathcal{P} i c(X) \rightarrow \mathcal{P} i c(X)$ satisfies $\left(\tilde{F}^{n}\right)^{*}=\left(\tilde{F}^{*}\right)^{n}$. Then 
Theorem 2. (See [12]) Let $F: P \mathbb{C}^{2} \rightarrow P \mathbb{C}^{2}$ be a birational map, $\tilde{F}$ be its regularized map and let $d_{n}=\operatorname{deg}\left(F^{n}\right)$. Then up to bimeromorphic conjugacy, exactly one of the following holds:

- The sequence $d_{n}$ grows quadratically, $\tilde{F}$ is an automorphism and $f$ preserves an elliptic fibration.

- The sequence $d_{n}$ grows linearly and $f$ preserves a rational fibration. In this case $\tilde{F}$ cannot be conjugated to an automorphism.

- The sequence $d_{n}$ is bounded, $\tilde{F}$ is an automorphism and $f$ preserves two generically transverse rational fibrations.

- The sequence $d_{n}$ grows exponentially.

In the first three cases $\delta(F)=1$ while in the last one $\delta(F)>1$. Furthermore in the first and second, the invariant fibrations are unique.

We recall that $f: \mathbb{C}^{2} \rightarrow \mathbb{C}$ preserves a fibration $V: \mathbb{C}^{2} \rightarrow \mathbb{C}$ if $f$ sends level curves of $V$ to level curves of $V$. If $f$ sends each level curve of $V$ to itself, it is said that $f$ is integrable and that $V$ is a first integral of $f$.

When the sequence $d_{n}$ is bounded it can happen that it is periodic or not. For mappings which are not periodic, we have the following result (Theorem A of [4]):

Theorem 3. (See [4]) Let $F: P \mathbb{C}^{2} \rightarrow P \mathbb{C}^{2}$ be a non-periodic birational map such that the corresponding sequence of degrees is bounded. Then $F$ is conjugate to an automorphism of $P \mathbb{C}^{2}$, which restricts to one of the following automorphisms on some open subset isomorphic to $\mathbb{C}^{2}$ :

(1) $(x, y) \mapsto(\alpha x, \beta y)$, where $\alpha, \beta \in \mathbb{C}^{*}$, and where the kernel of the group homomorphism $\mathbb{Z}^{2} \rightarrow \mathbb{C}^{*}$ given by $(i, j) \mapsto \alpha \beta^{j}$ is generated by $(k, 0)$ for some $k \in \mathbb{Z}$.

(2) $(x, y) \mapsto(\alpha x, y+1)$, where $\alpha \in \mathbb{C}^{*}$.

\subsection{Invariant fibrations}

From Theorem 2 we know the existence of rational invariant fibrations depending on the growth of $d_{n}$. To find them, we consider $V(x, y)=\frac{P(x, y)}{Q(x, y)}$ for some polynomials $P(x, y), Q(x, y)$ without common factors. If $V$ is an invariant fibration, then $f$ sends $V=k$ to $V=k^{\prime}$. In this work we consider that the relation between $k, k^{\prime}$ is of type $\psi(k)=\frac{\omega_{1} k+\omega_{2}}{\omega_{3} k+\omega_{4}}$ for some $\omega_{1}, \omega_{2}, \omega_{3}, \omega_{4} \in \mathbb{C}$. In particular we will have the following cases:

(a) $V(f)=V$, the integrable case. 
(b) $V(f)=\omega_{1} V$, the scaled fibration case.

(c) $V(f)=\omega_{1} V+\omega_{2}$, the scaled translated fibration case.

Note that in case $(a)$ in general the functions $P$ and $Q$ are invariant under $f$ as they satisfy the equation $P \cdot Q(f)=Q \cdot P(f)$ (unless that the denominators of $P(f)$ or $Q(f)$ are simplified with $Q$ or $P$ respectively). Similarly for case $(b)$ it follows. In case $(c)$ only $Q$ is invariant as it satisfies the relation $Q \cdot P(f)=\left(\omega_{1} P+\omega_{2} Q\right) \cdot Q(f)$.

Hence we always begin finding invariant algebraic curves. To find them, we introduce the following definition. Given a birational map and given a curve $C \subset P \mathbb{C}^{2}$ we define $F(C):=\overline{F(C \backslash \mathcal{I}(F)}$ to be the proper transform of $C$ by $F$. When $C \cap \mathcal{I}(F)=\emptyset$, we have that $\operatorname{deg} F(C)=d \cdot \operatorname{deg}(C)$ where $d$ is the degree of $F$. In general,

$$
\operatorname{deg} F(C)=d \cdot \operatorname{deg}(C)-\sum_{O \in \mathcal{I}(F)} m_{O}(C),
$$

where $m_{O}(C)$ is the algebraic multiplicity of $C$ at $O$ (see (1), pg. 416, [?]).

The approach is the following. Take an arbitrary curve $C$ and impose that $\operatorname{deg} F(C)=$ $\operatorname{deg} C$, that is, $(d-1) \operatorname{deg}(C)=\sum_{O \in \mathcal{I}(F)} m_{O}(C)$. For instance if $d=2$ and we consider $C$ of degree 3 , then a necessary condition for $C$ to be invariant under $f$ is that $C$ passes through three indeterminacy points of $F$ of multiplicity one or through one indeterminacy point with multiplicity two and another of multiplicity one or through one indeterminacy point with multiplicity three. In the first case, for instance, if $O_{1}, O_{2}, O_{3} \in \mathcal{I}(F)$, then there exist $T_{1}, T_{2}, T_{3} \in \mathcal{E}(F)$ such that $F^{-1}: T_{i} \rightarrow O_{i}$. Also, if $C=\{P=0\}$ for some polynomial $P$, then $F(C) \subset\left\{P \circ F^{-1}=0\right\}$ and we have that $P \circ F^{-1}=T_{1} \cdot T_{2} \cdot T_{3} \cdot \bar{P}$ for a certain polynomial $\bar{P}$, with $F(C)=\{\bar{P}=0\}$. Then imposing that $P-k \cdot \bar{P}=0$ we will find, if we get a solution, an invariant curve of degree three.

As we will see our particular mappings, sometimes depend on a number $\alpha$ which is a zero of certain polynomial $P$. Then all the calculations have to be made in $\frac{\mathbb{C}[\alpha]}{(P(\alpha))}[x, y]$, which in fact make them more complicated $\frac{\mathbb{C}[\alpha]}{(P(\alpha))}$ is the quotient ring $\mathbb{C}[\alpha]$ over the ideal generated by the polynomial $P(\alpha)$ ).

\section{The subfamily $\alpha_{2} \neq 0$.}

Taking into account that $\alpha_{1}, \beta_{1}$ and $\gamma_{2}$ are not zero, it can be proved that when $\alpha_{2} \neq 0$, after an affine change of coordinates $f(x, y)$ can be written as

$$
f(x, y)=\left(\alpha_{0}+\alpha_{1} x+y, \frac{x}{\gamma_{0}+y}\right) \quad, \quad \alpha_{1} \neq 0 .
$$


We consider the imbedding $(x, y) \mapsto[1: x: y] \in P \mathbb{C}^{2}$ into projective space and consider the induced map $F: P \mathbb{C}^{2} \rightarrow P \mathbb{C}^{2}$ given by:

$$
F\left[x_{0}: x_{1}: x_{2}\right]=\left[x_{0}\left(\gamma_{0} x_{0}+x_{2}\right):\left(\alpha_{0} x_{0}+\alpha_{1} x_{1}+x_{2}\right)\left(\gamma_{0} x_{0}+x_{2}\right): x_{0} x_{1}\right] .
$$

The indeterminacy locus of $F$ is $\mathcal{I}(F)=\left\{O_{0}, O_{1}, O_{2}\right\}$, where

$$
O_{0}=\left[1: 0:-\gamma_{0}\right], \quad O_{1}=\left[0: 1:-\alpha_{1}\right], \quad O_{2}=[0: 1: 0],
$$

and the indeterminacy locus of $F^{-1}$ is $\mathcal{I}\left(F^{-1}\right)=\left\{A_{0}, A_{1}, A_{2}\right\}$, where

$$
A_{0}=[0: 1: 0], \quad A_{1}=[0: 0: 1], \quad A_{2}=\left[-\alpha_{1}:-\alpha_{1}\left(\alpha_{0}-\gamma_{0}\right): 1\right] .
$$

The set of exceptional curves is given as $\mathcal{E}(F)=\left\{S_{0}, S_{1}, S_{2}\right\}$, where

$$
S_{0}=\left\{x_{0}=0\right\}, \quad S_{1}=\left\{\gamma_{0} x_{0}+x_{2}=0\right\}, \quad S_{2}=\left\{\gamma_{0} x_{0}+x_{2}+\alpha_{1} x_{1}=0\right\},
$$

and the set of exceptional curves of $F^{-1}$ is given as $\mathcal{E}\left(F^{-1}\right)=\left\{T_{0}, T_{1}, T_{2}\right\}$, where

$$
T_{0}=\left\{\left(\alpha_{0}-\gamma_{0}\right) x_{0}-x_{1}=0\right\}, \quad T_{1}=\left\{x_{0}-\alpha_{1} x_{2}\right\}, \quad T_{2}=\left\{x_{0}=0\right\} .
$$

Theorem 4. Let $F\left[x_{0}: x_{1}: x_{2}\right]$ be defined by

$$
F\left[x_{0}: x_{1}: x_{2}\right]=\left[x_{0}\left(\gamma_{0} x_{0}+x_{2}\right):\left(\alpha_{0} x_{0}+\alpha_{1} x_{1}+x_{2}\right)\left(\gamma_{0} x_{0}+x_{2}\right): x_{0} x_{1}\right]
$$

and let $\tilde{F}$ be the induced map after blowing up the point $A_{0}$. Then the following hold:

- If $\tilde{F}^{2 k}\left(A_{1}\right) \neq O_{1}$ for all $k \in \mathbb{N}$ and $\tilde{F}^{p}\left(A_{2}\right)=O_{0}$ for some $p \in \mathbb{N}$ then the characteristic polynomial associated with $F$ is given by

$$
\mathcal{X}_{p}=x^{p+1}\left(x^{2}-x-1\right)+x^{2},
$$

and

- for $p=0, p=1$ the sequence of degrees $d_{n}$ is bounded,

- for $p=2$ the sequence of degrees $d_{n}$ grows linearly,

- for $p>2$ the sequence of degrees $d_{n}$ grows exponentially.

- Assume that $\tilde{F}^{2 k}\left(A_{1}\right)=O_{1}$ for some $k \in \mathbb{N}$. Let $\tilde{F}_{1}$ be the induced map after we blow-up the points $A_{0}, A_{1}, \tilde{F}\left(A_{1}\right), \ldots, \tilde{F}^{2 k}\left(A_{1}\right)=O_{1}$. If $\tilde{F}_{1}^{p}\left(A_{2}\right) \neq O_{0}$ for all $p \in \mathbb{N}$, then the characteristic polynomial associated with $F$ is given by

$$
\mathcal{X}_{k}=x^{2 k+1}\left(x^{2}-x-1\right)+1,
$$

and the sequence of degrees grows exponentially. Furthermore $\delta(F) \rightarrow \delta^{*}$ as $k \rightarrow \infty$. 
- If $\tilde{F}^{2 k}\left(A_{1}\right)=O_{1}$ and $\tilde{F}^{p}\left(A_{2}\right)=O_{0}$ for some $p, k \in \mathbb{N}$ then the characteristic polynomial associated with $F$ is given by

$$
\mathcal{X}_{(k, p)}=x^{p+1}\left(x^{2 k+3}-x^{2 k+2}-x^{2 k+1}+1\right)+x^{2 k+3}-x^{2}-x+1,
$$

and

- for $p>\frac{2(1+k)}{k}$ the sequence of degrees $d_{n}$ grows exponentially.

- for $(k, p) \in\{(2,3),(1,4)\}$ the sequence of degrees $d_{n}$ either, it is periodic or it grows quadratically;

- for $(k, p) \in\{(k, 0),(k, 1),(k, 2),(1,3)\}$ the sequence of degrees $d_{n}$ is periodic.

- Assume that $\tilde{F}^{2 k}\left(A_{1}\right) \neq O_{1}$ and $\tilde{F}^{p}\left(A_{2}\right) \neq O_{0}$ for all $k, p \in \mathbb{N}$. Then the characteristic polynomial associated with $F$ is given by

$$
\mathcal{X}(x)=x^{2}-x-1,
$$

and the sequence of degrees grows exponentially with $\delta(F)=\delta^{*}$.

Proof. Observe that $S_{0} \rightarrow A_{0}=O_{2}$. The orbit of $A_{0}$ is SE. By blowing up $A_{0}$ we get the exceptional fibre $E_{0}$ and the new space $X$. The induced map $\tilde{F}: X \rightarrow X$ sends the curve $S_{0} \rightarrow E_{0} \rightarrow S_{0}$. Observe that now $\mathcal{I}(\tilde{F})=\left\{O_{0}, O_{1}\right\}$ and $\mathcal{E}(\tilde{F})=\left\{S_{1}, S_{2}\right\}$.

We see that $A_{1} \neq O_{1}$ and the exceptional curve $S_{1} \rightarrow A_{1} \in S_{0}$. We observe that the collision of orbits discussed in preliminaries is happening here. The orbit of $A_{1}$ under $\tilde{F}$ is as follows:

$$
S_{1} \rightarrow A_{1} \rightarrow\left[\gamma_{2}: \beta_{2}\right]_{E_{0}} \rightarrow\left[0: \alpha_{1}\left(\gamma_{0}+\beta_{2}\right): \beta_{1}\right] \in S_{0} \rightarrow \cdots
$$

After some iterates we can write the expression of $\tilde{F}^{2 k}\left(A_{1}\right)$ for all $k>0 \in \mathbb{N}$ as $\tilde{F}^{2 k}\left(A_{1}\right)=$ $\left[0: \alpha_{1}\left(\gamma_{0}+\beta_{2}\right)\left(1+\alpha_{1}+\alpha_{1}^{2}+\cdots+\alpha_{1}^{k-1}\right): \beta_{1}\right] \in S_{0}$. Observe that for some value of $k \in \mathbb{N}$ it is possible that $\tilde{F}^{2 k}\left(A_{1}\right)=O_{1}$. This happens when the following condition $k$ is satisfied for some $k$.

$$
\alpha_{1}^{2}\left(\gamma_{0}+\beta_{2}\right)\left(1+\alpha_{1}+\alpha_{1}^{2}+\cdots+\alpha_{1}^{k-1}\right)+\alpha_{2} \beta_{1}=0 .
$$

For such $k \in \mathbb{N}$ the orbit of $A_{1}$ is SE. By blowing up the points of this orbit we get the new space $X_{1}$ and the induced map $\tilde{F}_{1}$. Then under the action of $\tilde{F}_{1}$ we have

$$
S_{1} \rightarrow G_{0} \rightarrow G_{1} \rightarrow G_{2} \rightarrow \cdots \rightarrow G_{2 k-1} \rightarrow G_{2 k} \rightarrow T_{1}
$$

Then $\mathcal{I}\left(\tilde{F}_{1}\right)=\left\{O_{0}\right\}$ and $\mathcal{E}\left(\tilde{F}_{1}\right)=\left\{S_{2}\right\}$.

Now if the orbit of $A_{1}$ is $\mathrm{SE}$ and if $\tilde{F}_{1}^{p}\left(A_{2}\right)=O_{0}$ that is the orbit of $A_{2}$ is also SE for some $p \in \mathbb{N}$ then we have three $\mathrm{SE}$ orbits. If condition $k$ is not satisfied then with the 
extended map $\tilde{F}$ we have $\mathcal{I}(\tilde{F})=\left\{O_{0}, O_{1}\right\}$. Therefore we have two options: $\tilde{F}^{p}\left(A_{2}\right)=O_{0}$ or $\tilde{F}^{p}\left(A_{2}\right)=O_{1}$.

We claim that for all $p \in \mathbb{N}, \tilde{F}^{p}\left(A_{2}\right) \neq O_{1}$. Assume that $\tilde{F}^{p}\left(A_{2}\right)=O_{1}$ and assume that $F^{j}\left(A_{2}\right) \notin S_{0}$ for $j=1,2, \ldots, p-1 . \tilde{F}^{p}\left(A_{2}\right)=F^{p}\left(A_{2}\right)=O_{1}$. Since $O_{1} \in S_{0}$ and $A_{2} \notin S_{0}$ if $F^{p}\left(A_{2}\right)=O_{1}$ then $p$ would be greater than zero and since $S_{0}=T_{2}$, it would imply that $O_{1}=A_{1}$ or $O_{1}=A_{2}$, which is not the case (recall that the only points in $T_{2}$ which have a preimage are $A_{1}$ and $A_{2}$ ).

Contrarily, if it exists some $l \in \mathbb{N}, l<p$ such that $F^{j}\left(A_{2}\right) \notin S_{0}$ for $j=1,2, \ldots, l-1$ but $F^{l}\left(A_{2}\right) \in S_{0} \backslash\left\{O_{1}\right\}$ then $F^{l}\left(A_{2}\right)$ must be equal to $A_{1}$ or $A_{2}$ that is, $F^{l}\left(A_{2}\right)=A_{1}$ or $F^{l}\left(A_{2}\right)=A_{2}$. The second case is not possible as $A_{2}$ is a fixed point. In the first case $\tilde{F}^{p}\left(A_{2}\right)=\tilde{F}^{p-l}\left(F^{l}\left(A_{2}\right)\right)=\tilde{F}^{p-l}\left(A_{1}\right)=O_{1}$ which implies that $p=l+2 r$ and $\tilde{F}^{2 r}\left(A_{1}\right)=O_{1}$. Hence the orbit of $A_{1}$ must be SE and that condition $k$ must be satisfied for $k=r$ which is a contradiction. It implies that the only available possibility for $\mathcal{O}_{2}$ to be SE is to have that for some $p, \tilde{F}^{p}\left(A_{2}\right)=O_{0}$. After the blow up process we get

$$
S_{2} \rightarrow E_{1} \rightarrow E_{2} \rightarrow \cdots \rightarrow E_{p} \rightarrow E_{p+1} \rightarrow T_{0}
$$

The extended map $\tilde{F}_{2}$ is an automorphism when we have three SE orbits.

The above discussion gives us three different cases.

- One $S E$ orbit: This happens when $A_{0}=O_{2}$ with the conditions that $\tilde{F}^{2 k}\left(A_{1}\right) \neq O_{1}$ and $\tilde{F}^{p}\left(A_{2}\right) \neq O_{0}$ for all $k, p \in \mathbb{N}$. Therefore we have only one list $\mathcal{L}_{o}$ which is open that is $\mathcal{L}_{o}=\left\{\mathcal{O}_{0}=\left\{A_{0}=O_{2}\right\}\right\}$. By using Theorem 1 we find that $\delta(F)=\frac{\sqrt{5}+1}{2}$, which is given by the greatest root of the polynomial $X(x)=x^{2}-x-1$. Therefore it has exponential growth.

- Two $S E$ orbits $(a)$ : It is the case when $A_{0}=O_{2}, \tilde{F}^{p}\left(A_{2}\right)=O_{0}$ and $\tilde{F}^{2 k}\left(A_{1}\right) \neq O_{1}$ for all $k \in \mathbb{N}$. By organizing the orbits into lists we have one closed list $\mathcal{L}_{c}=\left\{\mathcal{O}_{0}=\right.$ $\left.\left\{A_{0}=O_{2}\right\}, \quad \mathcal{O}_{2}=\left\{A_{2}, \tilde{F}\left(A_{2}\right), \ldots, \tilde{F}^{p}\left(A_{2}\right)=O_{0}\right\}\right\}$. By utilizing Theorem 1 we find that the characteristic polynomial associated to $F$ is $\mathcal{X}_{p}=x^{p+1}\left(x^{2}-x-1\right)+x^{2}$. For $p=0$ and $p=1$ the sequence of degrees satisfies $d_{n+3}=d_{n}$ and $d_{n+4}=d_{n+3}$ respectively which corresponds towards boundedness of $d_{n}$.

For $p=2$ we get the polynomial $\mathcal{X}_{2}=x^{2}(x+1)(x-1)^{2}$. Looking at the first degrees we get that the sequence of degrees is $d_{n}=-1+2 n$.

For $p>2$, we observe that $\mathcal{X}_{p}(1)=0, \mathcal{X}^{\prime}{ }_{p}(1)=2-p<0$ and $\lim _{x \rightarrow+\infty} \mathcal{X}_{p}(x)=+\infty$. Hence $\mathcal{X}_{p}$ always has a root $\lambda>1$ and the result follows.

- Two $S E$ orbits $(b)$ : When we have $A_{0}=O_{2}, \tilde{F}_{1}{ }^{2 k}\left(A_{1}\right)=O_{1}$ and $\tilde{F}_{1}^{p}\left(A_{2}\right) \neq O_{0}$ for all $p \in \mathbb{N}$ then there is one open and one closed list and $\mathcal{X}_{k}=x^{2 k+1}\left(x^{2}-x-1\right)+1$. 
We observe that for all the values of $k \in \mathbb{N}, k \geq 1$ the polynomial $\mathcal{X}_{k}$ has always a root $\lambda>1$. Therefore $f$ has exponential growth.

- Three $S E$ orbits: In this case we have $A_{0}=O_{2}, \tilde{F}^{2 k}\left(A_{1}\right)=O_{1}, \tilde{F}^{p}\left(A_{2}\right)=O_{0}$, for a certain $p, k \in \mathbb{N}$. We have two closed lists as follows:

$$
\begin{gathered}
\mathcal{L}_{c}=\left\{\mathcal{O}_{0}=\left\{A_{0}=O_{2}\right\}, \quad \mathcal{O}_{2}=\left\{A_{2}, \tilde{F}\left(A_{2}\right), \ldots, \tilde{F}^{p}\left(A_{2}\right)=O_{0}\right\}\right\}, \\
\mathcal{L}_{c}=\left\{\mathcal{O}_{1}=\left\{A_{1}, \tilde{F}\left(A_{1}\right)_{E_{0}}, \ldots, \tilde{F}^{2 k}\left(A_{1}\right)_{S_{0}}=O_{1}\right\}\right\} .
\end{gathered}
$$

From Theorem 1 we can write $\mathcal{X}_{(k, p)}=x^{p+1}\left(x^{2 k+3}-x^{2 k+2}-x^{2 k+1}+1\right)+x^{2 k+3}-$ $x^{2}-x+1$. The map $\tilde{F}_{2}$ is an automorphism for all the values $(k, p)$. According to Diller and Favre in [12] the degree growth of iterates of an automorphism could be bounded, quadratic or exponential but it cannot be linear as in such a case the map is never an automorphism. For this we observe the behavior of $\mathcal{X}_{(k, p)}$ around $x=1$. We consider it's Taylor expansion near $x=1$ :

$$
\mathcal{X}_{(k, p)}(x)=2(2-k p+2 k)(x-1)^{2}+O\left(|x-1|^{3}\right) .
$$

Thus $\mathcal{X}_{(k, p)}$ vanishes at $x=1$ and has a maximum on it if $p>\frac{2(1+k)}{k}$. Since $\lim _{x \rightarrow+\infty \mathcal{X}_{(k, p)}(x)}=+\infty$, always exists a root greater than one. If $p \leq \frac{2(1+k)}{k}, k \geq 1$ then the pairs $(k, p)$ are in the set: $A_{(k, p)}=\{((k \geq 1), 0),((k \geq 1), 1),((k \geq$ 1), 2), $(1,3),(2,3),(1,4)\}$.

For $(k, p)=(k, 0), \mathcal{X}_{(k, 0)}(x)=\left(x^{2 k+2}-1\right)(x-1)(x+1)$, and hence the sequence of degrees is

$$
d_{n}=c_{0}+c_{1} n+c_{2}(-1)^{n}+c_{3}(-1)^{n} n+c_{4} \lambda_{1}^{n}+c_{5} \lambda_{2}^{n}+\ldots+c_{2 k+3} \lambda_{2 k}^{n},
$$

where $c_{i}$ are constants and $\lambda$ 's are the roots of polynomial $x^{2 k+2}=1$ different from \pm 1 . Since $\tilde{F}_{2}$ is an automorphism for all $(k, p)$, using [12] we have $c_{1}=0=c_{3}$. This implies that $d_{2 k+2+n}=d_{n}$, i. e., the sequence of degrees is periodic with period $2 k+2$. The argument for the proof of other values of $(k, p) \in A_{(k, p)}$ follows accordingly.

From the above theorem we see that zero entropy cases only appear when $\tilde{F}^{p}\left(A_{2}\right)=$ $O_{0}, \tilde{F}^{2 k}\left(A_{1}\right) \neq O_{1}$ for $p \in\{0,1,2\}$ and $\forall k \in \mathbb{N}$ and when $\tilde{F}^{p}\left(A_{2}\right)=O_{0}, \tilde{F}^{2 k}\left(A_{1}\right)=O_{1}$ for $(k, p) \in\{(k, 0),(k, 1),(k, 2),(1,3),(2,3),(1,4)\}$. We are going to study the dynamics of each case separately. Recall that condition $k$ is given by

$$
\alpha_{1}^{2} \gamma_{0}\left(1+\alpha_{1}+\alpha_{1}^{2}+\cdots+\alpha_{1}^{k-1}\right)+1=0
$$


The following proposition considers the case when $p=0$. From the above theorem we know that if condition $k$ is not satisfied the sequence $d_{n}$ is bounded and when it is satisfied, $d_{n}$ is a periodic sequence of period $2 k+2$. In any case we have to find two generically transverse fibrations. In the second case we present two first integrals functionally independent. We also prove that when $d_{n}$ is periodic, the mapping $f(x, y)$ is itself periodic.

Proposition 5. Assume that $A_{2}=O_{0}$. Then $f(x, y)$ can be written as

$$
f(x, y)=\left(\frac{1}{\alpha_{1}}+\alpha_{1} x+y, \frac{x}{\frac{1}{\alpha_{1}}+y}\right) \quad, \quad \alpha_{1} \neq 0
$$

and the following hold:

- If $\alpha_{1} \neq 1$ then $f(x, y)$ preserves the two generically transverse fibrations

$$
\begin{gathered}
V_{1}(x, y)=\frac{\sqrt{\alpha_{1}}-\alpha_{1}\left(\alpha_{1}+\sqrt{\alpha_{1}}\right) x+\alpha_{1}\left(1+2 \sqrt{\alpha_{1}}\right) y+\alpha_{1}^{2}\left(1+\sqrt{\alpha_{1}}\right) y^{2}}{1+\alpha_{1} y} \\
V_{2}(x, y)=\frac{-1+\alpha_{1}\left(1-\sqrt{\alpha_{1}}\right) x+\left(\sqrt{\alpha_{1}}-2 \alpha_{1}\right) y+\alpha_{1}\left(\sqrt{\alpha_{1}}-\alpha_{1}\right) y^{2}}{1+\alpha_{1} y}
\end{gathered}
$$

with $V_{1}(f(x, y))=-\sqrt{\alpha_{1}} V_{1}(x, y)$ and $V_{2}(f(x, y))=\sqrt{\alpha_{1}} V_{2}(x, y)$.

If $\alpha_{1}^{k+1}=1$ then $f$ is a $(2 k+2)$-periodic map. In this case $W_{1}(x, y)$ and $W_{2}(x, y)$ are two independent first integrals, where $W_{i}(x, y):=\left(V_{i}(x, y)\right)^{2 k+2}$.

- If $\alpha_{1}=1$ then $f(x, y)=\left(1+x+y, \frac{x}{1+y}\right)$ and it preserves the two generically transverse fibrations

$$
\begin{aligned}
& V_{1}(x, y)=\frac{1-2 x+3 y+2 y^{2}}{1+y} \\
& V_{2}(x, y)=\frac{1+2 x+3 y+2 y^{2}}{2(1+y)}
\end{aligned}
$$

with $V_{1}(f(x, y))=-V_{1}(x, y)$ and $V_{2}(f(x, y))=V_{2}(x, y)+1$. Furthermore $f(x, y)$ is integrable being $W(x, y)=V_{1}^{2}(x, y)$ a first integral.

Proof. Condition $A_{2}=O_{0}$ gives $\alpha_{0}=\gamma_{0}=\frac{1}{\alpha_{1}}$. From Theorem 4 we know that $f$ has two invariant fibrations. To find them follow the procedure explained in subsection 2.4. We consider an arbitrary cubic projective curve:

$$
\begin{aligned}
C\left[x_{0}: x_{1}: x_{2}\right]= & r_{0} x_{0}^{3}+r_{1} x_{0}^{2} x_{1}+r_{2} x_{0}^{2} x_{2}+r_{3} x_{0} x_{1}^{2}+r_{4} x_{0} x_{2}^{2} \\
& +r_{5} x_{0} x_{1} x_{2}+r_{6} x_{1}^{3}+r_{7} x_{1}^{2} x_{2}+r_{8} x_{1} x_{2}^{2}+r_{9} x_{2}^{3}
\end{aligned}
$$

and we force that $C$ is zero over the indeterminacy points of $F$, that is, $C\left(O_{0}\right)=C\left(O_{1}\right)=$ $C\left(O_{2}\right)=0$. Then

$$
C\left(F^{-1}\left[x_{0}: x_{1}: x_{2}\right]\right)=T_{0} \cdot T_{1} \cdot T_{2} \cdot \bar{C}\left[x_{0}: x_{1}: x_{2}\right],
$$


where $\left\{T_{0}, T_{1}, T_{2}\right\}=\mathcal{E}\left(F^{-1}\right)$ and $\bar{C}\left[x_{0}: x_{1}: x_{2}\right]$ is as follows:

$\left(r_{2} \alpha_{1}^{2}-2 r_{4} \alpha_{1}+3 r_{9}\right) x_{0}^{3}+\left(r_{4} \alpha_{1}^{2}-3 r_{9} \alpha_{1}\right) x_{0}^{2} x_{1}+\left(r_{2} \alpha_{1}^{3}+r_{1} \alpha_{1}^{2}-2 r_{4} \alpha_{1}^{2}-r_{5} \alpha_{1}+3 r_{9} \alpha_{1}+r_{8}\right) x_{0}^{2} x_{2}+$ $\left.\alpha_{1}^{2} r_{9} x_{0} x_{1}^{2}+\left(r_{5} \alpha_{1}\right] 2-2 r_{8} \alpha_{1}\right) x_{0} x_{1} x_{2}+\left(r_{1} \alpha_{1}^{3}-r_{5} \alpha_{1}^{2}+r_{8} \alpha_{1}\right) x_{0} x_{2}^{2}+\left(-r_{9} \alpha_{1}^{3}+r_{8} \alpha_{1}^{2}\right) x_{1}^{2} x_{2}+$ $\left(r_{9} \alpha_{1}^{3}+r_{3} \alpha_{1}^{2}-r_{3} \alpha_{1}^{2}\right) x_{1} x_{2}^{2}$.

The curve $\bar{C}$ is a degree three algebraic curve. We now impose that $C\left[x_{0}: x_{1}: x_{2}\right]=$ $k \bar{C}\left[x_{0}: x_{1}: x_{2}\right]$, then after some calculations we found (in affine coordinates)

$$
\begin{aligned}
& Q_{1}:=\sqrt{\alpha_{1}}-\alpha_{1}\left(\alpha_{1}+\sqrt{\alpha_{1}}\right) x+\alpha_{1}\left(1+2 \sqrt{\alpha_{1}}\right) y+\alpha_{1}^{2}\left(1+\sqrt{\alpha_{1}}\right) y^{2}, \\
& Q_{2}:=-1+\alpha_{1}\left(1-\sqrt{\alpha_{1}}\right) x+\left(\sqrt{\alpha_{1}}-2 \alpha_{1}\right) y+\alpha_{1}\left(\sqrt{\alpha_{1}}-\alpha_{1}\right) y^{2}, \\
& L:=1+\alpha_{1} y .
\end{aligned}
$$

The curves $Q_{1}$ and $Q_{2}$ are invariant algebraic curves while $L$ is an exceptional curve. Taking $V_{1}=Q_{1} / L$ and $V_{2}=Q_{2} / L$, simple computations prove that $V_{1}(f(x, y))=-\sqrt{\alpha_{1}} V_{1}(x, y)$, $V_{2}(f(x, y))=\sqrt{\alpha_{1}} V_{2}(x, y)$ and that $V_{1}(x, y), V_{2}(x, y)$ are generically transverse.

Now considering the mapping $\varphi(x, y):=\left(V_{1}(x, y), V_{2}(x, y)\right)$, we see that it is a birational mapping and it has the property that $\left(\varphi^{-1} \circ f \circ \varphi\right)(x, y)=\left(-\sqrt{\alpha_{1}} x, \sqrt{\alpha_{1}} y\right)$. From this we deduce that if $\alpha_{1}^{k+1}=1, \alpha_{1} \neq \pm 1$ then $f(x, y)$ is a $(2 k+2)$-periodic map. For $\alpha_{1}=-1$, $f$ is a 4-periodic map.Furthermore since $W_{i}(f(x, y))=W_{i}(x, y)$ for $i=1,2$ we get that $W_{1}(x, y), W_{2}(x, y)$ are first integrals.

When $\alpha_{1}=1$ we see that $V_{1}$ or $V_{2}$ is a constant function and that it is the unique value of the parameters which has this behaviour. If we take $\sqrt{1}=1$ we get the invariant fibration $V_{1}(x, y)=\frac{1-2 x+3 y+2 y^{2}}{1+y}$ with $V_{1}(f(x, y))=-V_{1}(x, y)$. To find $V_{2}$ we consider a rational function of type $V(x, y)=\frac{k_{0}+k_{1} x+k_{2} y+k_{3} y^{2}}{1+y}$ where $k_{i} \in \mathbb{C}$ for $i \in\{0,1,2,3\}$ and imposing $V(f(x, y))=V(x, y)+1$ after some calculations we find $V_{2}(x, y)$. Also in this case $f(x, y)$ is birationally conjugated to $(-x, y+1)$, see Theorem 3 again.

To deal with the case $p=1$, that is $F\left(A_{2}\right)=O_{0}$, we notice that this condition is equivalent to

$$
\alpha_{1}^{2}\left(\alpha_{0}-\gamma_{0}\right)+\alpha_{0} \alpha_{1}-1=0 \quad, \quad \alpha_{1} \gamma_{0}\left(\gamma_{0}-1\right)+\alpha_{0} \alpha_{1}-\gamma_{0}=0 \quad, \quad \gamma_{0} \alpha_{1}-1 \neq 0 .
$$

It is easy to see that it is true if and only if

$$
\gamma_{0}=\frac{1}{1+\alpha_{1}} \quad, \quad \alpha_{0}=\frac{1+\alpha_{1}+\alpha_{1}^{2}}{\alpha_{1}\left(1+\alpha_{1}\right)^{2}} \quad, \quad \alpha_{1} \notin\{0,-1\} .
$$

We note that for these maps condition (8) reads as $1+\alpha_{1}+\alpha_{1}^{2}+\cdots+\alpha_{1}^{k+1}=0$, which implies that $\alpha_{1}^{k+2}=1$.

Proposition 6. Assume that $F\left(A_{2}\right)=O_{0}$. then $f(x, y)$ can be written as:

$$
f(x, y)=\left(\frac{\alpha_{1}^{2}+\alpha_{1}+1}{\alpha_{1}\left(1+\alpha_{1}\right)^{2}}+\alpha_{1} x+y, \frac{x}{\frac{1}{1+\alpha_{1}}+y}\right) \quad, \quad \alpha_{1} \notin\{0,-1\}
$$


and

- If $\alpha_{1} \neq 1$ and $\alpha_{1}^{2}+\alpha_{1}+1 \neq 0$, then the map $f(x, y)$ preserves the two generically transverse fibrations

$$
\begin{aligned}
& V_{1}(x, y)=\frac{B_{0}+B_{1} x+B_{2} y+B_{3} y^{2}}{C_{0}+C_{1} x+C_{2} y+C_{3} x y+C_{4} y^{2}} \\
& V_{2}(x, y)=\frac{D_{0}+D_{1} x+D_{2} y+D_{3} y^{2}}{C_{0}+C_{1} x+C_{2} y+C_{3} x y+C_{4} y^{2}}
\end{aligned}
$$

where

$$
\begin{array}{ll}
B_{0}=\alpha_{1}{ }^{2}+\alpha_{1}+1 & C_{0}=\alpha_{1}{ }^{2}+\alpha_{1}+1 \\
B_{1}=-\left(1+\alpha_{1}\right)^{2}\left(\sqrt{\alpha_{1}}-\alpha_{1}-1\right) \sqrt{\alpha_{1}} & C_{1}=\alpha_{1}{ }^{2}\left(1+\alpha_{1}\right)^{2} \\
B_{2}=-\left(1+\alpha_{1}\right)\left(\alpha_{1}{ }^{\frac{3}{2}}-2 \alpha_{1}{ }^{2}-2 \alpha_{1}-1\right) & C_{2}=2 \alpha_{1}{ }^{3}+3 \alpha_{1}{ }^{2}+2 \alpha_{1}+1 \\
B_{3}=-\alpha_{1}\left(1+\alpha_{1}\right)^{2}\left(\sqrt{\alpha_{1}}-\alpha_{1}-1\right) & C_{3}=\alpha_{1}\left(\alpha_{1}-1\right)\left(1+\alpha_{1}\right)^{3} \\
& C_{4}=\alpha_{1}{ }^{2}\left(1+\alpha_{1}\right)^{2}
\end{array}
$$

and

$$
\begin{aligned}
& D_{0}=\alpha_{1}{ }^{2}+\alpha_{1}+1, \\
& D_{1}=-\left(1+\alpha_{1}\right)^{2}\left(\sqrt{\alpha_{1}}+\alpha_{1}+1\right) \sqrt{\alpha_{1}}, \\
& D_{2}=\left(1+\alpha_{1}\right)\left(\alpha_{1}^{3 / 2}+2 \alpha_{1}^{2}+2 \alpha_{1}+1\right), \\
& D_{3}=\alpha_{1}\left(1+\alpha_{1}\right)^{2}\left(\sqrt{\alpha_{1}}+\alpha_{1}+1\right) .
\end{aligned}
$$

with $V_{1}(f(x, y))=\frac{1}{\sqrt{\alpha_{1}}} V_{1}(x, y)$ and $V_{2}(f(x, y))=-\frac{1}{\sqrt{\alpha_{1}}} V_{2}(x, y)$.

If $1+\alpha_{1}+\alpha_{1}^{2}+\cdots+\alpha_{1}^{k+1}=0$ then $f(x, y)$ is a $2(k+2)$-periodic map. In this case $W_{i}(x, y)$ for $i \in\{1,2\}$ are two independent first integrals, where

$$
W_{i}(x, y):=V_{i}(x, y) \cdot V_{i}(f(x, y)) \cdot V_{i}\left(f^{2}(x, y)\right) \cdots V_{i}\left(f^{2 k+3}(x, y)\right)
$$

- If $\alpha_{1}=1$ then $f(x, y)$ preserves the two generically tranverse fibrations

$$
\begin{gathered}
V_{1}(x, y)=\frac{16 x y+4 x-6 y-3}{4 y^{2}+4 x+8 y+3} \\
V_{2}(x, y)=\frac{12 y^{2}-12 x+12 y+3}{4 y^{2}+4 x+8 y+3}
\end{gathered}
$$

with $V_{1}(f(x, y))=V_{1}(x, y)+1$ and $V_{2}(f(x, y))=-V_{2}(x, y)$. Hence $f(x, y)$ is integrable being $W(x, y)=V_{2}^{2}(x, y)$ a first integral.

- If $\alpha_{1}^{2}+\alpha_{1}+1=0$ then $f(x, y)$ is a 6-periodic mapping. It preserves the two generically tranverse fibrations

$$
V_{1}(x, y)=\frac{2 \alpha_{1}+2-x+\left(2 \alpha_{1}-1\right) y-\left(1+\alpha_{1}\right) y^{2}}{\left(\alpha_{1}+1\right) x+y+\left(\alpha_{1}-1\right) x y+\left(\alpha_{1}+1\right) y^{2}}
$$




$$
V_{2}(x, y)=\frac{\alpha_{1} x-\alpha_{1} y+y^{2}}{\left(\alpha_{1}+1\right) x+y+\left(\alpha_{1}-1\right) x y+\left(\alpha_{1}+1\right) y^{2}}
$$

with $V_{1}(f(x, y))=-\alpha_{1} V_{1}(x, y)$ and $V_{2}(f(x, y))=\alpha_{1} V_{2}(x, y)$. Furthermore $W_{1}(x, y):=$ $V_{1}^{6}(x, y)$ and $W_{2}(x, y):=V_{2}^{6}(x, y)$ are two independent first integrals.

Proof. From Theorem 4 we know that when $1+\alpha_{1}+\alpha_{1}^{2}+\cdots+\alpha_{1}^{k+1}=0, d_{n}$ is a periodic sequence while when $1+\alpha_{1}+\alpha_{1}^{2}+\cdots+\alpha_{1}^{k+1} \neq 0, d_{n}$ is bounded. In any case we have to find two generically transverse foliations.

We first search for invariant curves $C(x, y)=C_{0}+C_{1} x+C_{2} y+C_{3} x y+C_{4} y^{2}$. Then we consider a rational function $V(x, y)=\frac{P(x, y)}{C(x, y)}$, where $P(x, y)$ is a second degree polynomial. The imposition of condition $V(f(x, y))=k \cdot V(x, y)$ gives two invariant fibrations $V_{1}(x, y), V_{2}(x, y)$ for $k \in\left\{\frac{1}{\sqrt{\alpha_{1}}},-\frac{1}{\sqrt{\alpha_{1}}}\right\}$. Also we see that $V_{1}, V_{2}$ are generically transverse provided that $\alpha_{1} \neq \pm 1, \alpha_{1}^{2}+\alpha_{1}+1 \neq 0$.

Let $\varphi(x, y)$ be defined as $\varphi(x, y)=\left(V_{1}(x, y), V_{2}(x, y)\right)$. Then $\varphi(x, y)$ is a birational map and $\varphi^{-1} \circ f \circ \varphi$ gives the map $\left(\frac{1}{\sqrt{\alpha_{1}}} x,-\frac{1}{\sqrt{\alpha_{1}}} y\right)$. Hence if condition (8) is accomplished, i. e., if $1+\alpha_{1}+\alpha_{1}^{2}+\cdots+\alpha_{1}^{k+1}=0$, then $f(x, y)$ is a $(2 k+4)$-periodic map.

Now assume that $\alpha_{1}=1$. Substituting this value with $\sqrt{1}=1$ in the maps $V_{1}, V_{2}$ in the above paragraph we find that the first fibration is a constant function while the second one is $V_{2}(x, y)=\frac{12 y^{2}-12 x+12 y+3}{4 y^{2}+4 x+8 y+3}$, hence it satisfies $V_{2}(f(x, y))=-V_{2}(x, y)$. To find the other fibration $V(x, y)$ we consider a rational map with the same denominator of $V_{2}(x, y)$ and a degree two polynomial in the numerator and imposing $V(f(x, y))=V(x, y)+1$ we find the announced $V_{1}(x, y)$.

The fibrations when $\alpha_{1}^{2}+\alpha_{1}+1=0$ are encountered in a similar way.

Proposition 7. Assume that $F\left(F\left(A_{2}\right)\right)=O_{0}$. Then $f(x, y)$ can be written as

$$
f(x, y)=\left(\frac{\omega^{3}-\omega^{2}+1}{(\omega+1)\left(\omega^{2}-\omega+1\right)^{2}}+\omega^{2} x+y, \frac{\omega\left(\omega^{2}-\omega+1\right) x}{\omega-1+\left(\omega^{3}-\omega^{2}+\omega\right) y}\right), \omega(\omega+1)\left(\omega^{2}-\omega+1\right) \neq 0,
$$

and it preserves the fibration

$$
V(x, y)=\frac{B_{0}+B_{1} x+B_{2} y+B_{3} y^{2}}{\left(\omega+\left(\omega^{3}+1\right) y\right)\left(\omega-1+\left(\omega^{5}-\omega^{4}+\omega^{3}+\omega^{2}-\omega+1\right) x+\left(\omega^{3}-\omega^{2}+\omega\right) y\right)}
$$

where

$$
\begin{aligned}
& B_{0}=\left(\omega^{3}-\omega^{2}+1\right)(\omega-1), \\
& B_{1}=-\omega^{2}(\omega+1)\left(\omega^{2}-\omega+1\right)^{2}, \\
& B_{2}=\omega\left(\omega^{2}-\omega+1\right)\left(2 \omega^{3}-\omega^{2}-\omega+1\right), \\
& B_{3}=\omega^{3}(\omega+1)\left(\omega^{2}-\omega+1\right)^{2},
\end{aligned}
$$


with $V(f(x, y))=-\frac{1}{\omega} V(x, y)$. If $\omega^{4 k+6} \neq 1$ for all $k \in \mathbb{N}$ this fibration is unique. If $\omega^{m}=(-1)^{m}$ for some $m \in \mathbb{N}$, then $f(x, y)$ is integrable being $W(x, y)=V(x, y)^{m}$ a first integral.

When $\sum_{i=0}^{2 k+2}(-1)^{i} \omega^{i}=0$ for a certain $k \in \mathbb{N}$ then $f(x, y)$ is a $(4 k+6)$-periodic map.

Proof. Now we assume that $F^{2}\left(A_{2}\right)=O_{0}$. It is easy to see that it is equivalent to $\tilde{F}^{2}\left(A_{2}\right)=$ $O_{0}$. For the simplification of calculations we consider $\alpha_{1}=\omega^{2}$. It implies that the coefficients have to satisfy:

$$
\begin{aligned}
& E_{1}:=w^{6} \gamma_{0}^{2}-\left(\alpha_{0} w^{6}+\left(\alpha_{0}+1\right) w^{4}+\left(\alpha_{0}-2\right) w^{2}\right) \gamma_{0}+w^{4} \alpha_{0}+\alpha_{0}-1=0, \\
& E_{2}:=w^{4} \gamma_{0}^{3}-\left(w^{6}+w^{4}+w^{2}\right) \gamma_{0}^{2}+\left(\alpha_{0} w^{6}+\left(2 \alpha_{0}+1\right) w^{4}-w^{2}\right) \gamma_{0}-w^{4} \alpha_{0}-w^{2} \alpha_{0}+1=0 \\
& \gamma_{0} w^{2}-1 \neq 0 \quad, \quad w^{2} \gamma_{0}^{2}-\left(w^{2}+1\right) \gamma_{0}+w^{2} \alpha_{0} \neq 0 .
\end{aligned}
$$

Taking into account some resultants of $E_{1}$ and $E_{2}$ we find that the condition $F\left(F\left(A_{2}\right)\right)=$ $O_{0}$ gives the maps which appears in $(a)$. When $\omega^{2}-\omega+1=0$, that is, when $\alpha_{1}^{2}+\alpha_{1}+1=0$ we get the mappings $(b)$.

We note that for the parametric family $(a)$ condition $(8)$ is

$$
1-\omega+\omega^{2}-\omega^{3}+\omega^{4}+\cdots-\omega^{2 k+1}+\omega^{2 k+2}=0,
$$

which implies that $\omega$ is a $(4 k+6)$-root of unity, while for the two mappings $(b)$, condition $k$ never is satisfied.

Consider $f(x, y)$ that satisfies $(a)$. By looking for invariant curves we find that $V(x, y)$ can be written as shown in statement of $(a)$. A calculation shows that $V(f(x, y))=$ $-\frac{1}{\omega} V(x, y)$.

From this equality, we see that if $\omega^{m}=(-1)^{m}$ then $W(x, y):=V(x, y)^{m}$ is a first integral of $f(x, y)$.

If $\sum_{i=0}^{2 k+2}(-1)^{i} \omega^{i}=0$ for a certain $k \in \mathbb{N}$ then we know that the sequence of degrees is periodic of period $4 k+6$. We are going to prove that, the map itself is periodic of period $4 k+6$. Since $d_{4 k+6}=d_{0}=1$, the mapping $F^{4 k+6}$ is linear, that is:

$$
F^{4 k+6}\left[x_{0}: x_{1}: x_{2}\right]=\left[r_{0} x_{0}+r_{1} x_{1}+r_{2} x_{2}: p_{0} x_{0}+p_{1} x_{1}+p_{2} x_{2}: q_{0} x_{0}+q_{1} x_{1}+q_{2} x_{2}\right],
$$

for some constants $r_{i}, p_{i}, q_{i} \in \mathbb{R}$. As $S_{0}$ is invariant under the action $F^{2}$, it is invariant under the action of $F^{4 k+6}$ as well. This implies that we can write

$$
f^{4 k+6}(x, y)=\left(p_{0}+p_{1} x+p_{2} y, q_{0}+q_{1} x+q_{2} y\right),
$$

for some $p_{0}, p_{1}, p_{2}, q_{0}, q_{1}, q_{2} \in \mathbb{N}$. 
We find that the following two are the fixed points of $f$ and the third one is fixed by $f^{2}$.

$$
\begin{aligned}
& f i x_{1}=\left(\frac{1}{\left(\omega^{2}-\omega+1\right)(\omega+1)\left(\omega^{3}+1\right)},-\frac{\omega}{\omega^{3}+1}\right), \\
& f i x_{2}=\left(\frac{\omega^{3}-\omega^{2}+1}{\omega\left(\omega^{2}-\omega+1\right)\left(\omega^{2}-1\right)\left(\omega^{4}-\omega^{3}+\omega-1\right)},-\frac{\omega^{3}-\omega^{2}+1}{\omega\left(\omega^{4}-\omega^{3}+\omega-1\right)}\right), \\
& \text { fix }_{3}=\left(\frac{1}{\omega^{6}+2 \omega^{3}+1},-\frac{\omega}{\omega^{3}+1}\right) .
\end{aligned}
$$

Now these points must also be fixed by $f^{4 k+6}$. Then by finding the images of $f i x_{1}, f i x_{2}$ and $f i x_{3}$ under the action of $f^{4 k+6}$ using (11) such that $f^{4 k+6}\left(f i x_{1}\right)=f i x_{1}, f^{4 k+6}\left(f i x_{2}\right)=$ $f i x_{2}, f^{4 k+6}\left(f i x_{3}\right)=f i x_{3}$. Also as the sequence of degrees is periodic of period $4 k+6$ this implies that $\left(\tilde{F}_{1}^{*}\right)^{4 k+6}$ fixes the elements in the basis of Picard group. This implies that $\left(\tilde{F}_{1}^{*}\right)^{4 k+6}$ also fixes $E_{1}$ that is the blown up fibre at $A_{2}$. Then $F^{4 k+6}$ fixes the base point $A_{2}$ in $P \mathbb{C}^{2}$. By utilizing this information and then solving this system of four equations for the values of $p_{0}, p_{1}, p_{2}, q_{0}, q_{1}, q_{2}$ we find that $\left(p_{0}, p_{1}, p_{2}, q_{0}, q_{1}, q_{2}\right)=(0,1,0,0,0,1)$ which shows that $f^{4 k+6}(x, y)=(x, y)$.

Next case of zero entropy is when $p=3$ and $k=1$. Condition $k$ implies $\alpha_{1}^{2} \gamma_{0}+1=$ 0 , i. e., $\gamma_{0}=\frac{-1}{\alpha_{1}^{2}}$. It is easy to see that the condition $\tilde{F}^{3}\left(A_{2}\right)=O_{0}$ is equivalent to $F^{3}\left(A_{2}\right)=O_{0}$. Some computations show that it is true if and only if $\alpha_{1}^{6}+\alpha_{1}^{3}+1=0$ and $\alpha_{0}=-2 \alpha_{1}^{5}+\alpha_{1}^{3}-\alpha_{1}^{2}-\alpha_{1}$.

Proposition 8. Assume that $F^{3}\left(A_{2}\right)=O_{0}$ and that condition $k$ is satisfied for $k=1$. Then $f(x, y)$ can be written as

$$
f(x, y)=\left(-2 \alpha_{1}^{5}+\alpha_{1}^{3}-\alpha_{1}^{2}-\alpha_{1}+\alpha_{1} x+y, \frac{x}{\left(\alpha_{1}+\alpha_{1}^{4}\right)+y}\right), \alpha_{1}^{6}+\alpha_{1}^{3}+1=0,
$$

and it is a 18-periodic map. It preserves the two following generically transverse foliations $V_{1}(x, y)=\frac{H_{1}(x, y)}{C(x, y)^{2}}$ and $V_{2}(x, y)=\frac{H_{2}(x, y)}{C(x, y)^{2}}$ where

$$
C(x, y)=-\alpha_{1}{ }^{4}-\alpha_{1}{ }^{3}+\alpha_{1}{ }^{2}-2-\alpha_{1}{ }^{4} x+\left(\alpha_{1}{ }^{5}-2 \alpha_{1}{ }^{4}-\alpha_{1}{ }^{3}+2 \alpha_{1}{ }^{2}-\alpha_{1}-1\right) y-y^{2}
$$

and

$$
\begin{aligned}
H_{1}(x, y)= & A_{0}+A_{1} x+A_{2} y+A_{3} x^{2}+A_{4} x y+A_{5} y^{2}+A_{6} x^{2} y+A_{7} x y^{2}+A_{8} y^{3}+A_{9} x^{3} y+A_{10} x^{2} y^{2}+ \\
& A_{11} x y^{3}+A_{12} y^{4}+12 x^{3} y^{2}+A_{13} x^{2} y^{3}+A_{14} x y^{4}, \\
H_{2}(x, y)= & B_{0}+B_{1} x+B_{2} y+B_{3} x^{2}+B_{4} x y+B_{5} y^{2}+B_{6} x^{2} y+B_{7} x y^{2}+B_{8} y^{3}+3 \alpha_{1}^{4} x^{3} y+B_{9} x^{2} y^{2}+ \\
& B_{10} x y^{3}+B_{11} y^{4}+3 x^{2} y^{3}+B_{12} x y^{4},
\end{aligned}
$$


with

$$
\begin{aligned}
& A_{0}=31 \alpha_{1}{ }^{5}+23 \alpha_{1}{ }^{4}-23 \alpha_{1}{ }^{3}+35 \alpha_{1}+12 \\
& A_{8}=-4 \alpha_{1}{ }^{5}-16 \alpha_{1}{ }^{4}-4 \alpha_{1}{ }^{3}+10 \alpha_{1}{ }^{2}-6 \alpha_{1}-12 \\
& A_{1}=-18 \alpha_{1}{ }^{5}+4 \alpha_{1}{ }^{4}+20 \alpha_{1}{ }^{3}-14 \alpha_{1}{ }^{2}-14 \alpha_{1}+10 \\
& A_{9}=2 \alpha_{1}{ }^{4}+2 \alpha_{1}{ }^{3}+4 \alpha_{1}+4 \\
& A_{2}=32 \alpha_{1}{ }^{5}+48 \alpha_{1}{ }^{4}-14 \alpha_{1}{ }^{3}-20 \alpha_{1}{ }^{2}+44 \alpha_{1}+36 \\
& A_{3}=-3 \alpha_{1}{ }^{4}-2 \alpha_{1}{ }^{3}+2 \alpha_{1}{ }^{2}-2 \\
& A_{10}=12 \alpha_{1}{ }^{5}-12 \alpha_{1}{ }^{4}-12 \alpha_{1}{ }^{3}+18 \alpha_{1}{ }^{2}+6 \alpha_{1}-12 \\
& A_{4}=-60 \alpha_{1}{ }^{5}-4 \alpha_{1}{ }^{4}+58 \alpha_{1}{ }^{3}-34 \alpha_{1}{ }^{2}-48 \alpha_{1}+20 \\
& A_{5}=-6 \alpha_{1}^{5}+2 \alpha_{1}^{4}+8 \alpha_{1}^{3}-3 \alpha_{1}^{2}-2 \alpha_{1}+5 \\
& A_{11}=16 \alpha_{1}{ }^{5}+8 \alpha_{1}{ }^{4}-12 \alpha_{1}{ }^{3}+2 \alpha_{1}{ }^{2}+16 \alpha_{1}+6 \\
& A_{6}=8 \alpha_{1}{ }^{5}-18 \alpha_{1}{ }^{4}-16 \alpha_{1}{ }^{3}+16 \alpha_{1}{ }^{2}-20 \\
& A_{12}=16 \alpha_{1}{ }^{5}+8 \alpha_{1}{ }^{4}-12 \alpha_{1}{ }^{3}+2 \alpha_{1}{ }^{2}+16 \alpha_{1}+6 \\
& A_{13}=-16 \alpha_{1}{ }^{5}+2 \alpha_{1}{ }^{3}-14 \alpha_{1}{ }^{2}+4 \\
& A_{7}=-24 \alpha_{1}{ }^{5}-12 \alpha_{1}{ }^{4}+12 \alpha_{1}{ }^{3}-8 \alpha_{1}{ }^{2}-20 \alpha_{1} \\
& A_{14}=-4 \alpha_{1}{ }^{5}-4 \alpha_{1}{ }^{4}-2 \alpha_{1}{ }^{2}-2 \alpha_{1} \text {, } \\
& B_{0}=-38 \alpha_{1}{ }^{5}-20 \alpha_{1}{ }^{4}+31 \alpha_{1}{ }^{3}-7 \alpha_{1}{ }^{2}-40 \alpha_{1}-7 \\
& B_{1}=-11 \alpha_{1}{ }^{4}-4 \alpha_{1}{ }^{3}+9 \alpha_{1}{ }^{2}-4 \alpha_{1}-11 \\
& B_{2}=-62 \alpha_{1}{ }^{5}-51 \alpha_{1}{ }^{4}+44 \alpha_{1}{ }^{3}+5 \alpha_{1}{ }^{2}-72 \alpha_{1}-29 \\
& B_{3}=3 \alpha_{1}{ }^{5}-2 \alpha_{1}{ }^{3}+3 \alpha_{1}{ }^{2}+3 \alpha_{1}-1 \\
& B_{4}=31 \alpha_{1}{ }^{5}-15 \alpha_{1}{ }^{4}-33 \alpha_{1}{ }^{3}+32 \alpha_{1}{ }^{2}+21 \alpha_{1}-24 \\
& B_{5}=-25 \alpha_{1}{ }^{5}-20 \alpha_{1}{ }^{4}+19 \alpha_{1}{ }^{3}+\alpha_{1}{ }^{2}-28 \alpha_{1}-10 \\
& B_{6}=6 \alpha_{1}{ }^{5}+3 \alpha_{1}{ }^{4}-3 \alpha_{1}{ }^{3}+3 \alpha_{1}{ }^{2}+6 \alpha_{1}+6 \\
& B_{7}=20 \alpha_{1}{ }^{5}-30 \alpha_{1}{ }^{3}+16 \alpha_{1}{ }^{2}+24 \alpha_{1}-12 \\
& B_{8}=-3 \alpha_{1}{ }^{5}+7 \alpha_{1}{ }^{4}+3 \alpha_{1}{ }^{3}-9 \alpha_{1}{ }^{2}+2 \alpha_{1}+9 \\
& B_{9}=-3 \alpha_{1}{ }^{5}+6 \alpha_{1}{ }^{4}+6 \alpha_{1}{ }^{3}-6 \alpha_{1}{ }^{2}+3 \alpha_{1}+3 \\
& B_{10}=3 \alpha_{1}{ }^{5}-9 \alpha_{1}{ }^{4}+6 \alpha_{1}{ }^{2}-6 \alpha_{1}-3 \\
& B_{11}=3 \alpha_{1}^{5}+\alpha_{1}{ }^{4}-3 \alpha_{1}{ }^{3}+2 \alpha_{1} \\
& B_{12}=-3 \alpha_{1}{ }^{5}-3 \alpha_{1}{ }^{2} \text {. }
\end{aligned}
$$

They satisfy $V_{1}(f(x, y))=\alpha_{1}^{3} V_{1}(x, y)$ and $V_{2}(f(x, y))=\alpha_{1}^{2} V_{2}(x, y)$. Hence, $W_{1}(x, y)=$ $V_{1}(x, y)^{6}$ and $W_{2}=V_{2}(x, y)^{9}$ are two generically transverse first integrals of $f(x, y)$.

Proof. To find the foliations we began looking for degree 3 invariant curves. We only found $\bar{C}\left[x_{0}: x_{1}: x_{2}\right]=x_{0} C^{h}\left[x_{0}: x_{1}: x_{2}\right]$ where $C^{h}\left[x_{0}: x_{1}: x_{2}\right]$ is the homogeneous polynomial of degree two with $C^{h}\left[1: x_{1}: x_{2}\right]=C\left(x_{1}, x_{2}\right)$. Then we were looking for degree six invariant curves, with the condition that they passes trough the three indeterminacy points $O_{1}, O_{2}$ and $O_{3}$ with multiplicity two.Consequently, its image has also degree six. Forcing that this image coincides with the curve itself we found some of them. For instance, the two numerators of $V_{1}(x, y)$ and $V_{2}(x, y)$. A computation gives that $V_{1}(f(x, y))=\alpha_{1}^{3} V_{1}(x, y)$, $V_{2}(f(x, y))=\alpha_{1}^{2} V_{2}(x, y)$ and that they are generically transverse. Clearly $W_{1}(x, y)$ and $W_{2}(x, y)$ are first integrals of $f(x, y)$ because $\alpha_{1}^{18}=1$.

From Theorem 4 we know that the sequence of degrees is periodic of periodic 18. To prove that the map is periodic we apply the result of [7], which says that if a map has two independent first integrals, then it is a periodic map.

Proposition 9. Assume that $F^{3}\left(A_{2}\right)=O_{0}$ and that condition $k$ is satisfied for $k=2$. Then either:

(a) There exists $\alpha_{1}$ with $\alpha_{1}^{4}+\alpha_{1}^{3}+\alpha_{1}^{2}+\alpha_{1}+1=0$ such that $f(x, y)$ is of the form

$$
f(x, y)=\left(-\left(\alpha_{1}^{3}+2 \alpha_{1}^{2}+\alpha_{1}+2\right)+\alpha_{1} x+y, \frac{x}{-\left(1+\alpha_{1}^{2}+\alpha_{1}^{3}\right)+y}\right) .
$$


That map $f(x, y)$ preserves the elliptic fibration $V(x, y)=\frac{L(x, y) \cdot P(x, y) \cdot Q(x, y)}{R(x, y)^{2}}$ where

$$
\begin{aligned}
& L(x, y)=\left(-\alpha_{1}^{3}-2 \alpha_{1}^{2}-2 \alpha_{1}-2+\left(\alpha_{1}^{2}+\alpha_{1}\right) x+y\right) \\
& P(x, y)=\left(y x+\left(-\alpha_{1}^{2}-1\right) x+\alpha_{1}^{2} y+\alpha_{1}^{3}+\alpha_{1}\right) \\
& Q(x, y)=\left(\alpha_{1}{ }^{3} y^{2}+\left(-\alpha_{1}{ }^{3}-\alpha_{1}{ }^{2}-\alpha_{1}-1\right) x y+\left(-\alpha_{1}{ }^{3}+\alpha_{1}{ }^{2}\right) y+\alpha_{1}\right) \\
& R(x, y)=\left(y^{2}-\left(3 \alpha_{1}{ }^{3}+3 \alpha_{1}{ }^{2}+2 \alpha_{1}+2\right) y-x \alpha_{1}{ }^{2}+\alpha_{1}{ }^{3}-\alpha_{1}{ }^{2}+1\right)
\end{aligned}
$$

with $V(f(x, y))=\alpha_{1}^{2} V(x, y)$ and this fibration is unique. Furthermore $f$ is integrable being $W(x, y)=V(x, y)^{5}$ a first integral of $f$.

(b) The map $f(x, y)$ is:

$$
f(x, y)=\left(\frac{1}{4}+x+y, \frac{x}{-\frac{1}{2}+y}\right) .
$$

That map $f(x, y)$ preserves the elliptic fibration $V(x, y)=$

$$
\frac{256 x^{3} y^{2}+384 x^{2} y^{3}+128 x y^{4}+128 x^{3} y+192 x^{2} y^{2}+32 x y^{3}-16 y^{4}-16 x^{2}-8 x y+8 y^{2}-8 x-1}{\left(-4 y^{2}+4 x+1\right)^{2}}
$$

with $V(f(x, y))=V(x, y)$ and this fibration is unique. Hence $f$ is integrable.

Proof. When $k=2$ condition $k$ says $\alpha_{1}^{2} \gamma_{0}\left(1+\alpha_{1}\right)+1=0$, i. e., $\gamma_{0}=\frac{-1}{\alpha_{1}^{2}\left(1+\alpha_{1}\right)}$. Also here $\tilde{F}^{3}\left(A_{2}\right)=O_{0}$ is equivalent to $F^{3}\left(A_{2}\right)=O_{0}$. Some tedious computations show that it is true if and only if either, $1+\alpha_{1}+\alpha_{1}^{2}+\alpha_{1}^{3}+\alpha_{1}^{4}=0$ with $\alpha_{0}=-\left(\alpha_{1}^{3}+2 \alpha_{1}^{2}+\alpha_{1}+2\right)$ or $\alpha_{1}=1$ with $\alpha_{0}=\frac{1}{4}$.

For the mappings $(a)$ we find the invariant conic:

$y^{2}-\left(3 \alpha_{1}^{3}+3 \alpha_{1}^{2}+2 \alpha_{1}+2\right) y-\alpha_{1}^{2} x+\alpha_{1}^{3}-\alpha_{1}^{2}+1$ and a degree five invariant curve, the one given by $L(x, y) \cdot P(x, y) \cdot Q(x, y)=0$. Taking the quotient of them, some calculations prove that in fact $V(f(x, y))=\alpha_{1}^{2} V(x, y)$.

To prove the uniqueness of the invariant fibration we have to see that $d_{n}$ is not a periodic sequence. Assume that it is, i. e., assume that $d_{n}$ is 30 -periodic. Then $F^{30}$ has degree one:

$$
F^{30}\left[x_{0}: x_{1}: x_{2}\right]=\left[r_{0} x_{0}+r_{1} x_{1}+r_{2} x_{2}: p_{0} x_{0}+p_{1} x_{1}+p_{2} x_{2}: q_{0} x_{0}+q_{1} x_{1}+q_{2} x_{2}\right] .
$$

As before, since $S_{0}$ is invariant under $F^{2}$, we can write $f^{30}$ as follows:

$$
f^{30}(x, y)=\left(p_{0}+p_{1} x+p_{2} y, q_{0}+q_{1} x+q_{2} y\right) .
$$

Now, using that the conic $y^{2}-\left(3 \alpha_{1}^{3}+3 \alpha_{1}^{2}+2 \alpha_{1}+2\right) y-x \alpha_{1}^{2}+\alpha_{1}^{3}-\alpha_{1}^{2}+1=0$ must be invariant under $f^{30}$ and that the point $\left(-\alpha_{1}^{3}-\alpha_{1}^{2}, 1\right)$ (which is a fixed point for $f$ ) must also be fixed for $f^{30}$, after some calculations we get that either, $f^{30}$ is the identity or $f^{30} \circ f^{30}$ is the identity. In any case, it would imply that $f$ is a periodic mapping.

But we claim that the mapping $f$ itself is not periodic. If it were the case, then $f^{k}(x, y)=$ $(x, y)$ for some $k$ multiple of 30 . We observe that $f$ sends:

$$
\{L(x, y)=0\} \longrightarrow\{P(x, y)=0\} \longrightarrow\{Q(x, y)=0\} \longrightarrow\{L(x, y)=0\} .
$$


In particular $f^{3}$ sends $\{Q(x, y)=0\}$ to $\{Q(x, y)=0\}$. We see that the curve $\{Q(x, y)=0\}$ can be parameterized by $y$, because $\{Q(x, y)=0\}$ if and only if $x=\varphi(y):=\frac{\alpha_{1}\left(\alpha_{1}^{2}+1+\alpha_{1} y\right)}{\alpha_{1}^{2}+1-y}$. Then $f^{3}(\varphi(y), y)=(\varphi(h(y)), h(y))$ where $h(y)=\frac{u(y)}{v(y)}$ with

$$
\begin{aligned}
u(y)= & -5 \alpha_{1}{ }^{3}-3 \alpha_{1}{ }^{2}-\alpha_{1}-6+\left(29 \alpha_{1}{ }^{3}+10 \alpha_{1}{ }^{2}+13 \alpha_{1}+27\right) y+\left(-54 \alpha_{1}{ }^{3}-3 \alpha_{1}{ }^{2}-31 \alpha_{1}-40\right) y^{2}+ \\
& \left(50 \alpha_{1}{ }^{3}-9 \alpha_{1}{ }^{2}+32 \alpha_{1}+20\right) y^{3}+\left(-22 \alpha_{1}{ }^{3}+7 \alpha_{1}{ }^{2}-23 \alpha_{1}-3\right) y^{4}+\left(2 \alpha_{1}{ }^{3}-6 \alpha_{1}{ }^{2}+5 \alpha_{1}-2\right) y^{5}
\end{aligned}
$$

and

$$
\begin{aligned}
v(y)= & \left(5 \alpha_{1}{ }^{3}-\alpha_{1}{ }^{2}+4 \alpha_{1}+2\right) y+\left(-11 \alpha_{1}{ }^{3}+8 \alpha_{1}{ }^{2}-11 \alpha_{1}\right) y^{2}+\left(9 \alpha_{1}{ }^{3}-12 \alpha_{1}{ }^{2}+12 \alpha_{1}-9\right) y^{3}+ \\
& \left(8 \alpha_{1}{ }^{2}-9 \alpha_{1}+8\right) y^{4}+\left(-3 \alpha_{1}{ }^{3}-6 \alpha_{1}{ }^{2}-\alpha_{1}-5\right) y^{5} .
\end{aligned}
$$

If $f$ where a periodic mapping, $h$ also would be periodic. But $h$ has the fixed point $\bar{y}=1+\alpha_{1}^{2}$ and the derivative of $h(y)$ at this points gives zero. And it is a contradiction because periodic maps have the eigenvalues of modulus one at the fixed points.

To prove $(b)$ we begin by proving that the sequence of degrees grows quadratically. Then the prescribed fibration will be unique. The characteristic polynomial associated to $d_{n}$ is $(x+1)\left(x^{2}+x+1\right)\left(x^{4}+x^{3}+x^{2}+x+1\right)(x-1)^{4}$ which implies that either, $d_{n}$ grows quadratically or it is periodic. It only depends on the initial conditions, that is on the values of $d_{n}$ for $n=1,2, \ldots, 11$. For that mapping we have been able to calculate these numbers: $2,3,5,8,12,16,22,28,35,43,52$ which implies that

$$
d_{n}=\frac{97}{72}+\frac{5 n^{2}}{12}-\frac{1}{8}(-1)^{n}-\frac{1}{9}\left(\frac{-1+\sqrt{3} I}{2}\right)^{n}-\frac{1}{9}\left(\frac{-1-\sqrt{3} I}{2}\right)^{n},
$$

that is, $d_{n}$ grows quadratically.

To find $V(x, y)$ we searched for invariant curves and we found one of degree two: $-4 y^{2}+$ $4 x+1$ and one of degree five, the numerator of $V(x, y)$. Taking the quotient of them, we verified that it satisfies $V(f(x, y))=V(x, y)$.

The last class with zero entropy is when $p=4$ with $k=1$. The condition $k=1$ says that $\gamma_{0}=\frac{-1}{a 1^{2}}$. From the proof and notations of Theorem 4 we know that:

$$
\begin{aligned}
& S_{0} \longrightarrow E_{0} \longrightarrow S_{0}=T_{2}, \\
& S_{1} \longrightarrow G_{0} \longrightarrow G_{1} \longrightarrow G_{2} \longrightarrow T_{1}, \\
& S_{2} \longrightarrow E_{1} \longrightarrow E_{2} \longrightarrow E_{3} \longrightarrow E_{4} \longrightarrow E_{5} \longrightarrow T_{0} .
\end{aligned}
$$

Hence, if $A_{2} \in S_{1}$, i.e., $\alpha_{1}=-1$, then it could happen that $\tilde{F}^{4}\left(A_{2}\right)=O_{0}$. Following the orbit of $A_{2}$ we get: $\tilde{F}\left(A_{2}\right)=[1: 0]_{G_{0}}, \tilde{F}^{2}[1: 0]_{G_{0}}=\left[1:-\alpha_{0}\right]_{G_{2}}$ and $\tilde{F}\left[1:-\alpha_{0}\right]_{G_{2}}=[1: 0:$ $1]=O_{0}$. Hence we see that $\tilde{F}^{4}\left(A_{2}\right)=O_{0}$ for all values of $\alpha_{0}$, provided that $\alpha_{1}=-1$ and $\gamma_{0}=\frac{-1}{a 1^{2}}=-1$. 
Proposition 10. Assume that $\tilde{F}^{4}\left(A_{2}\right)=O_{0}$ and that condition $k$ is satisfied for $k=1$, where $\tilde{F}$ is the mapping induced by $F$ after blowing up the point $[0: 1: 0]$.

Then either:

(a) The map $f(x, y)$ can be written as

$$
f(x, y)=\left(\alpha_{0}-x+y, \frac{x}{y-1}\right)
$$

and it preserves the unique elliptic fibration

$$
V(x, y)=\frac{\alpha_{0} x y-x^{2} y+x y^{2}}{y-1}
$$

with $V(f(x, y))=V(x, y)$. Hence $f$ is integrable.

(b) The map $f(x, y)$ can be written as

$$
f(x, y)=\left(x+y, \frac{x}{y-1}\right)
$$

and it preserves the unique elliptic fibration

$$
V(x, y)=\frac{-2 y^{2}+2 x+y+1}{x y(x+y)}
$$

with $V(f(x, y))=-V(x, y)$. Furthermore $f$ is integrable, being $W(x, y)=V(x, y)^{2} a$ first integral of $f$.

(c) The map $f(x, y)$ can be written as

$$
f(x, y)=\left(\alpha_{1} x+y, \frac{x}{y+1}\right) \text { with } \alpha_{1}^{2}+1=0
$$

and it preserves the unique elliptic fibration

$$
V(x, y)=-\frac{x y\left(\alpha_{1} y-x\right)}{\left(\alpha_{1} y+\alpha_{1}-2 x-y-1\right)\left(-1+\alpha_{1}-2 y\right)}
$$

with $V(f(x, y))=\alpha_{1} V(x, y)$. Furthermore $f$ is integrable, being $W(x, y)=V(x, y)^{4} a$ first integral of $f$.

(d) The map $f(x, y)$ can be written as

$$
f(x, y)=\left(1-\alpha_{1}^{3}+\alpha_{1} x+y, \frac{x}{\alpha_{1}^{2}+y}\right) \text { with } \alpha_{1}^{4}+1=0
$$

and it preserves the unic elliptic fibration

$$
V(x, y)=-\frac{Q_{1}(x, y) Q_{2}(x, y) Q_{3}(x, y)}{\left(\alpha_{1}{ }^{3}-1+\left(\alpha_{1}{ }^{2}+1\right) x+\left(\alpha_{1}{ }^{2}+\alpha_{1}\right) y\right)^{2}\left(\alpha_{1}{ }^{3}-1+\alpha_{1}{ }^{2}\left(\alpha_{1}{ }^{2}+1\right) y\right)^{2}}
$$


where

$$
\begin{aligned}
& Q_{1}(x, y)=\alpha_{1}^{2}+2 \alpha_{1}+1+\left(2 \alpha_{1}^{2}+\alpha_{1}+1\right) y+\alpha_{1}^{3} y^{2}-x y, \\
& Q_{2}(x, y)=2 \alpha_{1}{ }^{3}+\alpha_{1}{ }^{2}-1+\left(-2 \alpha_{1}{ }^{3}+\alpha_{1}+1\right) x+\left(\alpha_{1}{ }^{2}+2 \alpha_{1}+1\right) y+\alpha_{1}{ }^{3} x^{2}+\alpha_{1}{ }^{2} x y, \\
& Q_{3}(x, y)=-\left(\alpha_{1}{ }^{2}+2 \alpha_{1}+1\right)+\left(2 \alpha_{1}{ }^{3}+\alpha_{1}{ }^{2}-1\right) y-\alpha_{1}{ }^{2} x y .
\end{aligned}
$$

with $V(f(x, y))=\alpha_{1}^{2} V(x, y)$. Furthermore $f$ is integrable, being $W(x, y)=V(x, y)^{4} a$ first integral of $f$.

Proof. The mapping (a) corresponds to the case $\alpha_{1}=-1$ and $\gamma_{0}=-1$ when there is collisions of orbits. Looking at the expression of $F^{4}\left(A_{2}\right)$ and after tedious computations we get that $F^{4}\left(A_{2}\right)=O_{0}$ if and only if $f(x, y)$ is one of $(b),(c)$ or $(d)$.

To see the uniqueness of the fibrations we have to prove that $d_{n}$ grows quadratically. The characteristic polynomial associated to $d_{n}$ is $(x-1)^{4}(x+1)^{2}\left(x^{2}+1\right)\left(x^{2}+x+1\right)$ which implies that either, $d_{n}$ grows quadratically or it is periodic. It only depends on the initial conditions, that is on the values of $d_{n}$ for $n=1,2, \ldots, 10$. For each one of the mappings which appear in the statement, we have been able to calculate these numbers. In the four cases they give $2,3,5,7,11,15,20,25,32,39$, which implies that

$$
d_{n}=\frac{23}{16}+\frac{3}{8} n^{2}-\frac{3}{16}(-1)^{n}-\frac{1}{8} I^{n}-\frac{1}{8}(-I)^{n} .
$$

In order to prove $(a)$ we find the family of invariant curves $\lambda\left(\alpha_{0} x y-x^{2} y+x y^{2}\right)+\mu(y-$ $1)=0$. Then taking $V=\frac{P}{Q}$ with and $P=\alpha_{0} x y-x^{2} y+x y^{2}$ and $Q=y-1$ we have that $V(f(x, y))=V(x, y)$.

To prove $(b)$ we easily see that

$$
\{x=0\} \longrightarrow\{y=0\} \longrightarrow\{x+y=0\} \longrightarrow\{x=0\}
$$

and hence $x y(y+x)$ is an invariant cubic. Then taking $V$ as the quotient of a conic and the invariant cubic and imposing $V(f(x, y))=k V(x, y)$ we found that the conic can be taken as $-2 y^{2}+2 x+y+1$ and $k=-1$.

To prove $(c)$ we find that the straight line $\alpha_{1} y+\alpha_{1}-2 x-y-1=0$ is sent to the straight line $-1+\alpha_{1}-2 y=0$ and viceversa, which implies that their product is an invariant curve of degree two. Also it can be seen that

$$
\{x=0\} \longrightarrow\{y=0\} \longrightarrow\left\{\alpha_{1} y-x=0\right\} \longrightarrow\{x=0\}
$$

and hence $x y\left(\alpha_{1} y-x\right)$ is an invariant cubic. Taking $V$ as the quotient of this invariant curves we get that $V(f(x, y))=\alpha_{1} V(x, y)$ and the result follows

To see $(d)$ we began searching invariant curves of degree three and we found (in projective coordinates)

$C\left[x_{0}: x_{1}: x_{2}\right]=x_{0} \cdot\left(\left(\alpha^{3}-1\right) x_{0}+\left(\alpha^{2}+1\right) x_{1}+\left(\alpha^{2}+\alpha\right) x_{2}\right) \cdot\left(\left(\alpha^{3}-1\right) x_{0}+\alpha^{2}\left(\alpha^{2}+1\right) x_{2}\right)$ 
Now we take a conic that passes through two indeterminacy points of $F$, and we impose that its image (a conic again) also passes through two indeterminacy points of $F$. This gives a third conic which we impose to be equal to the first one. With this we find $Q_{1}, Q_{2}, Q_{3}$. Then $Q_{1} \cdot Q_{2} \cdot Q_{3}$ is an invariant curve of degree six. Taking $V$ as the quotient of $Q_{1} \cdot Q_{2} \cdot Q_{3}$ over $C[1: x: y]^{2}$ we get that $V(f(x, y))=\alpha_{1}^{2} V(x, y)$.

We can now state the main theorem of this section:

Theorem 11. Assume that

$$
f(x, y)=\left(\alpha_{0}+\alpha_{1} x+\alpha_{2} y, \frac{\beta_{0}+\beta_{1} x+\beta_{2} y}{\gamma_{0}+\gamma_{2} y}\right), \alpha_{1} \neq 0, \beta_{1} \neq 0, \gamma_{2} \neq 0, \alpha_{2} \neq 0 .
$$

Then it has zero entropy if and only if after an affine change of coordinates it can be written as one of the mappings which appear in the statements of Propositions 5, 6, 7, 8, 9, 10. Each one of them has the invariant fibrations which are stated in the above propositions.

\section{The subfamily $\alpha_{2}=0$.}

By conjugating $f(x, y)$ via $h(x, y)=\left(\beta_{1} \gamma_{2} x-\frac{\beta_{0} \gamma_{2}-\beta_{2} \gamma_{0}}{\beta_{1} \gamma_{2}}, \beta_{1} y-\frac{\gamma_{0}}{\gamma_{2}}\right)$ and renaiming the parameters, we can consider

$$
f(x, y)=\left(\alpha_{0}+\alpha_{1} x, \frac{x+\beta_{2} y}{y}\right) \text { with } \alpha_{1} \neq 0 .
$$

We consider the induced map in the projective plane $: F: P \mathbb{C}^{2} \rightarrow P \mathbb{C}^{2}$ given by

$$
F\left[x_{0}: x_{1}: x_{2}\right]=\left[x_{0} x_{2}:\left(\alpha_{0} x_{0}+\alpha_{1} x_{1}\right) x_{2}: x_{0}\left(x_{1}+\beta_{2} x_{2}\right)\right]
$$

The indeterminacy sets of $F$ and $F^{-1}$ are $\mathcal{I}(F)=\left\{O_{0}, O_{1}, O_{2}\right\}$, where

$$
O_{0}=[1: 0: 0], \quad O_{1}=[0: 0: 1], \quad O_{2}=[0: 1: 0],
$$

and $\mathcal{I}\left(F^{-1}\right)=\left\{A_{0}, A_{1}, A_{2}\right\}$, where

$$
A_{0}=[0: 1: 0], \quad A_{1}=[0: 0: 1], \quad A_{2}=\left[1: \alpha_{0}: \beta_{2}\right] .
$$

Furthermore the exceptional curves of $F$ and $F^{-1}$ are the following:

$$
\begin{gathered}
S_{0}=\left\{x_{0}=0\right\}, \quad S_{1}=\left\{x_{2}=0\right\}, \quad S_{2}=\left\{x_{1}=0\right\}, \\
T_{0}=\left\{\alpha_{0} x_{0}-x_{1}=0\right\}, \quad T_{1}=\left\{\beta_{2} x_{0}-x_{2}=0\right\}, \quad T_{2}=\left\{x_{0}=0\right\} .
\end{gathered}
$$


Theorem 12. Let $f(x, y)$ be a map of type (18) with $\gamma_{2} \neq 0, \alpha_{1} \neq 0, \beta_{1} \neq 0$ and suppose that $\alpha_{2}=0$. If $f^{p}\left(\alpha_{0}, \beta_{2}\right)=(0,0)$ for some $p \in \mathbb{N}$ then the characteristic polynomial associated with $f$ is given by

$$
\mathcal{X}_{p}=\left(x^{p+1}+1\right)(x-1)^{2}(x+1),
$$

and the sequence of degrees of $f$ is periodic with period $2 p+2$. If no such $p$ exists then the characteristic polynomial associated with $f$ is

$$
\mathcal{X}=(x-1)^{2}(x+1),
$$

and the sequence of degrees $d_{n}$ grows linearly.

Proof. Observe that $S_{0} \rightarrow A_{0}=O_{2}$ and $S_{1} \rightarrow A_{1}=O_{1}$. Hence we blow up the points $A_{0}, A_{1}$ getting the exceptional fibres $E_{0}, E_{1}$. Let $X$ be the new space and let $\tilde{F}: X \rightarrow X$ be the corresponding map on $X$. Then the map $\tilde{F}$ sends the curve $S_{0} \rightarrow E_{0} \rightarrow S_{0}$ and $S_{1} \rightarrow E_{1} \rightarrow T_{1}$. We observe that no new indeterminacy points are created therefore $\mathcal{I}(\tilde{F})=$ $\left\{O_{0}\right\}$ and $\mathcal{E}(\tilde{F})=\left\{S_{2}\right\}$.

Assume that there exists $p \in \mathbb{N}$ such that $\tilde{F}^{p}\left(A_{2}\right)=O_{0}$. Then we blow up $A_{2}, \tilde{F}\left(A_{2}\right)$, $\tilde{F}^{2}\left(A_{2}\right), \ldots, \tilde{F}^{p}\left(A_{2}\right)=O_{0}$ getting the exceptional fibres which we call $E_{2}, E_{3}, \ldots, E_{p+2}$. Set $\tilde{F}_{1}: X_{1} \rightarrow X_{1}$ the extended map. Performing the blow up at $O_{0}$, since $T_{0}$ is sent to $O_{0}$ via $F^{-1}$, we have that $\tilde{F}_{1}^{-1}: T_{0} \rightarrow E_{p+2}$. Then $S_{2} \rightarrow E_{2} \rightarrow E_{3} \rightarrow \cdots \rightarrow E_{p+1} \rightarrow E_{p+2} \rightarrow T_{0}$. Hence $\tilde{F}_{1}: X_{1} \rightarrow X_{1}$ is an AS map and also an automorphism. Taking into account that $A_{2}=\left[1: \alpha_{0}: \beta_{2}\right]$ and $O_{0}=[1: 0: 0]$ belong to the affine plane, it is clear that condition $\tilde{F}^{p}\left(A_{2}\right)=O_{0}$ reads as $f^{p}\left(\alpha_{0}, \beta_{2}\right)=(0,0)$.

Now we have two closed lists as follows

$$
\begin{gathered}
\mathcal{L}_{c_{1}}=\left\{\mathcal{O}_{0}=\left\{A_{0}=O_{2}\right\}, \quad \mathcal{O}_{2}=\left\{A_{2}, \tilde{F}\left(A_{2}\right), \ldots, \tilde{F}^{p}\left(A_{2}\right)=O_{0}\right\}\right\}, \\
\mathcal{L}_{c_{2}}=\left\{\mathcal{O}_{1}=\left\{A_{1}=O_{1}\right\}\right\} .
\end{gathered}
$$

Then by using Theorem 1 we find that the characteristic polynomial associated to $F$ is

$$
\mathcal{X}=\left(x^{p+1}+1\right)(x-1)^{2}(x+1)
$$

If $p$ is even then $x^{p+1}+1$ has the factor $x+1$ and $\mathcal{X}=(x-1)^{2}(x+1)^{2}\left(x^{p}-x^{p-1}+\cdots-x+1\right)$. Hence the sequence of degrees is $d_{n}=c_{0}+c_{1} n+c_{2}(-1)^{n}+c_{3} n(-1)^{n}+c_{4} \lambda_{1}^{n}+c_{5} \lambda_{2}^{n}+\ldots+$ $c_{p+3} \lambda_{p}^{n}$, where $c_{i}$ are constants and $\lambda_{1}, \lambda_{2}, \ldots, \lambda_{p}$ are the roots of polynomial $x^{p}-x^{p-1}+$ $\cdots-x+1$. By looking at $d_{n}$ we see that $f$ does not grow quadratically or exponentially. As our map $\tilde{F}_{1}$ is an automorphism then by using the results from Diller and Favre in [12] we see that also cannot have linear growth. Therefore we must have $c_{1}=c_{3}=0$. Hence the 
sequence of degrees must be periodic. This implies that $d_{2 p+2+n}=d_{n}$ i.e. the sequence of degrees is periodic with period $2 p+2$. If $p$ is odd then $d_{n}$ is also periodic of period $2 p+2$.

If $\tilde{F}^{p}\left(A_{2}\right) \neq O_{0}$ for all $p \in \mathbb{N}$, then we have two lists which are open and closed as follows:

$$
\mathcal{L}_{o}=\left\{\mathcal{O}_{0}=\left\{A_{0}=O_{2}\right\}\right\} \quad, \quad \mathcal{L}_{c}=\left\{\mathcal{O}_{1}=\left\{A_{1}=O_{1}\right\}\right\} .
$$

Then $\delta(F)$ is determined by the polynomial $(x-1)^{2}(x+1)$, and $\delta(f)=1$. The sequence of degrees is $d_{n}=\frac{5}{4}+\frac{1}{2} n-\frac{1}{4}(-1)^{n}$.

Theorem 13. Let $f(x, y)=\left(\alpha_{0}+\alpha_{1} x, \frac{x+\beta_{2} y}{y}\right)$ with $\alpha_{1} \neq 0$ and set $h(x)=\alpha_{0}+\alpha_{1} x$. Then the following hold:

1. If $f^{p}\left(\alpha_{0}, \beta_{2}\right) \neq(0,0)$ for all $p \in \mathbb{N}$ then $f$ preserves the fibration $V_{1}(x, y)=x$ with $V_{1}(f(x, y))=\alpha_{0}+\alpha_{1} V_{1}(x, y)$, and this fibration is unique. If $\alpha_{1}^{n}=1$ for some $n>1, \alpha_{1} \neq 1$, the map is integrable being

$$
W(x, y)=x \cdot h(x) \cdot h(h(x)) \cdots h^{n-1}(x)
$$

a first integral of $f$. Also when $\alpha_{1}=1$ and $\alpha_{0}=0, f$ is integrable.

2. If $f^{p}\left(\alpha_{0}, \beta_{2}\right)=(0,0)$ for some $p \geq 1$, then $f$ is a $(2 p+2)$-periodic map. These maps have $W(x, y)=x \cdot h(x) \cdot h(h(x)) \cdots h^{2 p+1}(x)$ as a first integral.

3. If $\left(\alpha_{0}, \beta_{2}\right)=(0,0)$, then $f(x, y)=\left(\alpha_{1} x, \frac{x}{y}\right)$ and it preserves the two generically transverse fibrations

$$
V_{1}(x, y)=\sqrt{\alpha_{1}} y+\frac{x}{y} \quad, \quad V_{2}(x, y)=-\sqrt{\alpha_{1}} y+\frac{x}{y}
$$

with $V_{1}(f(x, y))=\sqrt{\alpha_{1}} V_{1}(x, y)$ and $V_{2}(f(x, y))=-\sqrt{\alpha_{1}} V_{2}(x, y)$. When $\alpha_{1}^{n}=1$ for some $n$ then $f$ is $2 n$-periodic and $W_{1}(x, y)=V_{1}(x, y)^{2 n}, W_{2}(x, y)=V_{2}(x, y)^{2 n}$ are two independent first integrals.

Remark 14. We notice that when $p=0$, that is, $\alpha_{0}=0=\beta_{2}$, then $\varphi(x, y):=$ $=\left(V_{1}(x, y), V_{2}(x, y)\right)$ is a birational map. It turns out that using $\varphi(x, y)$ as a conjugation we get the map $\left(\sqrt{\alpha_{1}} x,-\sqrt{\alpha_{1}} y\right)$. These result on linearizations was already pointed out on the work of Blanc and Deserti, see[4]. Furthermore, the sequence of degrees is $d_{n}=2,1,2,1,2,1, \ldots$ a two-periodic sequence, and avoiding the case $\alpha_{1}^{n}=1$ for some $n$, the map itself in not more periodic.

For $p \geq 1$ the map is periodic and hence it has two independent first integrals. There is a method to find them (see [7]). For instance, when $\alpha_{1}=-1$ and $\alpha_{0}=-\beta_{2}^{2} \quad($ case $p=1)$ i.e., $f(x, y)=\left(-x-\beta_{2}^{2}, \frac{x+\beta_{2} y}{y}\right)$, we have that

$$
H(x, y)=y+\frac{x+\beta_{2} y}{y}+\frac{x\left(\beta_{2}-y\right)}{x+\beta_{2} y}+\frac{x+\beta_{2}^{2}}{\beta_{2}-y}
$$


is a first integral of $f$ and $W(x, y), H(x, y)$ are generically transverse.

Proof. If $f^{p}\left(\alpha_{0}, \beta_{2}\right) \neq(0,0)$ for all $p \in \mathbb{N}$ then from the above theorem we know that $d_{n}$ grows linearly, and hence we know that $f(x, y)$ has a unique invariant fibration. Clearly $V_{1}(x, y)=x$ is an invariant fibration and when $\alpha_{1}=1$ and $\alpha_{0}=0, V_{1}(x, y)$ is a first integral. When $\alpha_{1}^{n}=1$ the function $h(x)$ is periodic of period $n$ and hence $W(x, y)$ is a first integral of $f(x, y)$.

Now assume that $f^{p}\left(\alpha_{0}, \beta_{2}\right)=(0,0)$ for a certain $p \in \mathbb{N}$. From Theorem (12) we know that the sequence of degrees $d_{n}$ is $2 p+2$ periodic. We are going to see that $f(x, y)$ itself is a periodic map. Since the map $F^{2 p+2}$ is linear, we can consider that for some constants $r_{i}, p_{i}, q_{i} \in \mathbb{R}$ the map $F^{2 p+2}$ can be written in the following form:

$$
F^{2 p+2}\left[x_{0}: x_{1}: x_{2}\right]=\left[r_{0} x_{0}+r_{1} x_{1}+r_{2} x_{2}: p_{0} x_{0}+p_{1} x_{1}+p_{2} x_{2}: q_{0} x_{0}+q_{1} x_{1}+q_{2} x_{2}\right] .
$$

We know that $S_{0}$ is invariant under the action $F^{2}$ therefore it is invariant under the action of $F^{2 p+2}$ as well. This implies that

$$
F^{2 p+2}\left[0: x_{1}: x_{2}\right]=\left[0: x_{1}: x_{2}\right],
$$

which further implies that $r_{1} x_{1}+r_{2} x_{2}=0$ for all complex numbers $x_{1}, x_{2}$. This is only possible if $r_{1}=r_{2}=0$. Then we can write

$$
F^{2 p+2}\left[x_{0}: x_{1}: x_{2}\right]=\left[x_{0}: \frac{p_{0}}{r_{0}} x_{0}+\frac{p_{1}}{r_{0}} x_{1}+\frac{p_{2}}{r_{0}} x_{2}: \frac{q_{0}}{r_{0}} x_{0}+\frac{q_{1}}{r_{0}} x_{1}+\frac{q_{2}}{r_{0}} x_{2}\right],
$$

which in the affine plane by taking $x_{0}=1$ and rewriting the parameters, as new parameters, the function $F^{2 p+2}$ can be written as following:

$$
f^{2 p+2}(x, y)=\left(p_{0}+p_{1} x+p_{2} y, q_{0}+q_{1} x+q_{2} y\right),
$$

for any $p_{0}, p_{1}, p_{2}, q_{0}, q_{1}, q_{2} \in \mathbb{R}$. We find that the following two points are fixed for $f(x, y)$ :

$$
(X, \pm Y)=\left(\frac{\alpha_{0}}{1-\alpha_{1}}, \frac{\beta_{2}\left(1-\alpha_{1}\right) \pm \sqrt{\left(1-\alpha_{1}\right)^{2} \beta_{2}^{2}+4 \alpha_{0}\left(1-\alpha_{1}\right)}}{2\left(1-\alpha_{1}\right)}\right),
$$

As these points are fixed by $f$ so they are also fixed points of $f^{2 p+2}$. Then finding their images under the action of $f^{2 p+2}$ using (20) we get a system equations such that $f^{2 p+2}(X, Y)[1]=X, f^{2 p+2}(X, Y)[2]=Y, f^{2 p+2}(X,-Y)[2]=-Y$. Also as the sequence of degrees is periodic of period $2 p+2$ this implies that $\left(\tilde{F}_{1}^{*}\right)^{2 p+2}$ fixes the elements in the basis of Picard group. This implies that $\left(\tilde{F}_{1}^{*}\right)^{2 p+2}$ also fixes $E_{2}$ that is the blown up fiber at $A_{2}$. Then $F^{2 p+2}$ fixes the base point $A_{2}$ in $P \mathbb{C}^{2}$. By utilizing this information and then solving the system of equations for the values of $p_{0}, p_{1}, p_{2}, q_{0}, q_{1}, q_{2}$ we find that $\left(p_{0}, p_{1}, p_{2}, q_{0}, q_{1}, q_{2}\right)=(0,1,0,0,0,1)$ which implies that $f^{2 p+2}(x, y)=(x, y)$. 
Finally, $p=0$ that is when $A_{2}=O_{0}$, by iterating the function $f(x, y)=\left(\alpha_{1} x, \frac{x}{y}\right)$ we find that $f^{2 n}(x, y)=\left(\alpha_{1}^{2 n} x, \alpha_{1}^{n} y\right)$ and $f^{2 n+1}(x, y)=\left(\alpha_{1}^{2 n+1} x, \alpha_{1}^{n} \frac{x}{y}\right)$. Now observe that for $\alpha_{1}^{n}=$ 1 we have $f^{2 n}(x, y)=(x, y)$ and $f^{2 n+1}(x, y)=\left(x, \frac{x}{y}\right)$. Therefore $f$ is $2 n$-periodic. Now for $\alpha_{1}^{n} \neq 1$ through simple calculations we find that $f$ preserves the announced fibrations $V_{1}(x, y)$ and $V_{2}(x, y)$.

\section{Acknowledgements}

The first author is supported by Ministry of Economy, Industry and Competitiveness of the Spanish Government through grant MINECO/FEDER MTM2016-7 7278-P and also by the grant 2014-SGR-568 from AGAUR, Generalitat de Catalunya.

\section{References}

[1] Bedford, E. and Kim, K. On the degree growth of birational mappings in higher dimension, J. Geom. Anal. 14 (2004), 567-596.

[2] Bedford, E. and Kim, K. Periodicities in Linear Fractional Recurrences: Degree Growth of Birational Surface Maps, Michigan Math. J. 54 (2006), 647-670.

[3] Bischi, G.I., Gardini, L. and Mira, C. Plane maps with denominator I: Some generic properties, Internat. J. Bifur. Chaos Appl. Sci. Engrg. 9 (1999), 119-153.

[4] Blanc, J. and Deserti, J. Degree growth of birational maps of the plane, Ann. Sc. Norm. Super. Pisa Cl. Sci. 5, 14 (2015), 507-533.

[5] Cima, A., Gasull, A. and Mañosas, F. On Periodic Rational Difference Equations of Order k, J. Difference Equ. Appl., 10(6), (2004), 549-559.

[6] Cima, A., Gasull, A. and Mañosa, V. Dynamics of some rational discrete dynamical systems via invariants, J. of Bif. and Ch., 16(3), (2006), 631-645.

[7] Cima, A., Gasull, A. and V. Mañosa. Global periodicity and complete integrability of discrete dynamical systems, J. Difference Equations 12, 7, (2006), 697-716.

[8] Cima, A., Gasull, A. and Mañosa, V. Non-autonomous 2-periodic Gumovski-Mira difference equations, Internat. J. Bifur. Chaos Appl. Sci. Engrg., 22(11), (2012), 1250264 (14 pages).

[9] Csornyei, M. and Laczkovich, M. Some Periodic and Non-periodic Recursions, Monatsh. Math., 132, (2001), 215-236. 
[10] Cima, A. and Zafar, S. Dynamical classification of a Family of Birational Maps of the plane via algebraic entropy. arXiv:1704.07731

[11] Cima, A. and Zafar, S. Zero entropy for some Birational Maps of the plane. ArXiv: 1704.07108

[12] J. Diller and C. Favre. Dynamics of bimeromorphic maps of surfaces, Amer. J. Math. 123 (2001), 1135-1169.

[13] Diller, J. Dynamics of Birational Maps of $P \mathbb{C}^{2}$, Indiana Univ. Math. J. 45, 3, (1996), $721-772$.

[14] Fornaes, J-E and Sibony, N. Complex dynamics in higher dimension. II, Modern methods in complex analysis (Princeton, NJ, 1992), 135182, Ann. of Math. Stud., 137, Princeton Univ. Press, Princeton, NJ, 1995. Michigan Math. J. 54 (2006), 647-670.

[15] Iatrou, A. and Roberts, J. Integrable mappings of the plane preserving biquadratic invariant curves, J. Phys. A 34, 34, (2001), 6617-6636.

[16] Jogia, D and Roberts, J. Birational maps that send biquadratic curves to biquadratic curves, J. Phys. A 48, 8, (2015), 08FT02, 13 pp.

[17] Ladas, G. On the rational recursive sequence $x_{n+1}=\frac{a+\beta x_{n}+x_{n-1}}{A+B x_{n}+C x_{n-1}}$, J. Differ. Equ.Appl., 1, (1995) 317-321.

[18] Ladas, G., Gibbons, C.H. and Kulenovic, M.R.S. On the rational recursive sequence $x_{n+1}=\frac{\alpha+\beta x_{n}+\gamma x_{n-1}}{A+B x_{n}}$. In: Proceedings of the Fifth International Conference on Difference Equations and Applications, Temuco, Chile, 37 January 2000, pp. 141-158. Taylor and Francis, London (2002).

[19] Ladas, G. Kulenovic, M. and Prokup, N. On the Recursive Sequence $x_{n+1}=\frac{a x_{n}+b x_{n-1}}{A+x_{n}}$, Journal of Difference Equations and Applications, 6 , 563-576, (2000).

[20] Ladas, G., Kulenovic, M.R.S, Martins, L.F. and Rodrigues, I.W. On the Dynamics of $x_{n+1}=\frac{a+b x_{n}}{A+B x_{n}+C x_{n-1}}$, Facts and Conjectures, Computers and Mathematics with Applications , 45, (2003), 1087-1099.

[21] Zayed, E.M.E. and El-Moneam, M.A. On the rational recursive sequence $x_{n+1}=$ $\frac{a x_{n}+b x_{n-k}}{c x n-d x_{n-k}}$. Commun. Appl. Nonlinear Anal. 15, (2008), 67-76. 